ARTICLE

Received 11 Jun 2014 | Accepted 25 Feb 2015 | Published 17 Apr $2015 \quad$ DOl: 10.1038/ncomms7764

\title{
Mir-17-92 regulates bone marrow homing of plasma cells and production of immunoglobulin G2C
}

Shengli $\mathrm{Xu}^{1,2, \star}, \mathrm{Xijun} \mathrm{Ou}^{1, \star}$, Jianxin Huo ${ }^{1}$, Kristen $\mathrm{Lim}^{1}$, Yuhan Huang ${ }^{1}$, Sheena Chee ${ }^{1}$ \& Kong-Peng Lam ${ }^{1,2,3}$

The polycistronic mir-17-92 cluster, also known as oncomir-1, was previously shown to be essential for early B lymphopoiesis. However, its role in late-stage B-cell differentiation and function remains unexplored. Here we ablate mir-17-92 in mature B cells and demonstrate that mir-17-92 is dispensable for conventional B-cell development in the periphery. Interestingly, mir-17-92-deficiency in B cells leads to enhanced homing of plasma cells to the bone marrow during T-cell-dependent immune response and selectively impairs IgG2c production. Mechanistically, mir-17-92 directly represses the expression of Sphingosine 1-phosphate receptor 1 and transcription factor IKAROS, which are, respectively, important for plasma cell homing and IgG2c production. We further show that deletion of mir-17-92 could reduce $\operatorname{lgG} 2 \mathrm{c}$ anti-DNA autoantibody production and hence mitigate immune complex glomerulonephritis in Shp1-deficient mice prone to autoimmunity. Our results identify important roles for mir-17-92 in the regulation of peripheral B-cell function.

\footnotetext{
${ }^{1}$ Bioprocessing Technology Institute, Agency for Science, Technology and Research (A-STAR), Singapore 138668. ${ }^{2}$ Department of Physiology, National University of Singapore, Singapore, Singapore 117597. ${ }^{3}$ Department of Microbiology, National University of Singapore, Singapore 117545. * These authors contributed equally to this work. Correspondence and requests for materials should be addressed to S.X. (email: xu_shengli@bti.a-star.edu.sg) or to K.-P.L. (email: lam_kong_peng@bti.a-star.edu.sg).
} 
$\mathrm{B}$ cells and antibodies are central elements of adaptive humoral immunity and critical for host protection. B lymphopoiesis begins with committed haematopoietic stem cells differentiating into pro-B, pre-B and immature $\mathrm{B}$ cells in the bone marrow (BM) and cumulates with mature $B$ cells seeding the peripheral lymphoid organs. On activation by foreign antigens, naive $B$ cells undergo terminal differentiation into memory $\mathrm{B}$ and antibodysecreting plasma cells (PCs) that provide life-long protection ${ }^{1,2}$. B-cell differentiation is governed by a network of transcription factors $^{3,4}$. Recently, microRNAs (miRNAs) are shown to exert additional control over B-cell differentiation and function ${ }^{5,6}$.

MiRNAs are endogenously expressed short non-coding RNAs that fine-tune gene expression at the post-transcriptional level by either promoting the degradation or impeding the translation of target mRNAs ${ }^{7-9}$. A 'seed region' of six to eight nucleotides at the $5^{\prime}$ end of a miRNA determines its target specificity ${ }^{10}$. The importance of miRNAs in B-cell biology is highlighted by the drastic defects in mice with global deletion of miRNAs caused by ablating Dicer, a RNAse III endonuclease critical for miRNA processing, in precursor ${ }^{11}$, peripheral mature ${ }^{12}$ and activated ${ }^{13} \mathrm{~B}$ cells. When Dicer was deleted at the earliest stage of B-cell maturation using mb-1 Cre, B lymphopoiesis was severely blocked at the pro-B to pre-B-cell transition ${ }^{11}$. This defect was mainly attributed to the absence of mir-17-92 whose seed sequences were enriched in the $3^{\prime}$-untranslated regions ( $3^{\prime}$ UTRs) of mRNAs upregulated in Dicer-deficient pro-B cells ${ }^{11}$.

MiR-17-92, or oncomir-1, is a polycistronic miRNA cluster that yields six mature miRNAs, including mir-17, mir-18a, mir-19a, mir-20a, mir-19b and miR-92a. It was first identified as a potent miRNA oncogene on human chromosome 13q31, a region that was amplified in a number of B-cell malignancies ${ }^{14,15}$. Overexpression of mir-17-92 in haematopoietic cells significantly accelerated c-Myc induced B-lymphomagenesis in mice $^{16}$ and B-cell-specific mir-17-92 transgenic mice developed lymphoma with high penetrance ${ }^{17}$. On the other hand, germline deletion of mir-17-92 compromised early B lymphopoiesis suggesting that mir-17-92 plays essential roles in early B-cell development ${ }^{18}$. Pro-apoptotic BIM was identified as a direct target of mir-17-92 and its upregulation in mutant pro-B cells accounted for the impairment in B-lymphopoiesis ${ }^{18}$.

In this study, we deleted mir-17-92 in mature B cells using CD19-Cre to examine its role in late-stage B-cell differentiation and function. In contrast to its essential role in early $B$ lymphopoiesis in the BM, mir-17-92-deficency does not perturb conventional B-cell populations in the periphery. Interestingly, during T-cell-dependent (TD) antibody response, there is increased PCs in the BM due to elevated expression of Sphingosine 1-phosphate receptor 1 (S1PR1), which mediates $\mathrm{BM}$ homing of PCs ${ }^{19}$. We show that five out of six members of mir-17-92 cluster directly target the $3^{\prime} \mathrm{UTR}$ of S1pr1 and removal of one S1pr1 allele could rebalance S1pr1 expression and normalize the enhanced BM homing phenotype of mir-17-92deficient PCs. We also reveal that immunoglobulin G2c (IgG2c) production is impaired in the absence of mir-17-92 and this defect is due to increased expression of the transcription factor IKAROS, which is important for IgG2c production ${ }^{20}$ and directly repressed by mir-92a (ref. 21). Furthermore, we demonstrate that mir-17-92 deletion in B cells could reduce IgG2c autoantibody production and attenuate immune complex glomerulonephritis in autoimmune Shp1-deficient mice. Taken together, our findings identify novel roles for mir-17-92 in regulating peripheral B-cell function by fine-tuning different target genes.

\section{Results}

B-cell development in mice lacking mir-17-92 in B cells. To address the role of mir-17-92 in peripheral B cells, we deleted
loxP-flanked (floxed) mir-17-92 alleles in mice using CD19-Cre that is inefficient with floxed gene deletion in early B cells but mediates efficient gene deletion in mature $B$ lymphocytes ${ }^{22}$. This allows us to bypass the pro-B to pre-B-cell block in mir-17-92 germline-deleted mice ${ }^{18}$ to investigate its role in the later stages of $\mathrm{B}$-cell maturation and function.

Fluorescence-activated sorting (FACS) analyses revealed that the frequency and numbers of pro-B $\left(\mathrm{B} 220^{+} \mathrm{CD} 43^{+} \mathrm{IgM}^{-}\right)$and pre-B $\left(\mathrm{B} 220^{+} \mathrm{CD}_{4} 3^{-} \mathrm{IgM}^{-}\right)$cells were comparable in the $\mathrm{BM}$ of

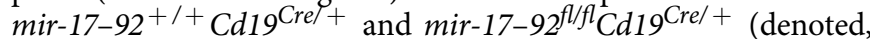
respectively, as mir-17-92 control and mir-17-92 bKO thereafter; Fig. 1a, Supplementary Fig. 1a). Quantitative reverse trancription-PCR (qRT-PCR) analyses indicated that the expression of individual mir-17-92 member was not significantly reduced in $\mathrm{BM} \mathrm{B}_{2} 20^{+} \mathrm{IgM}^{-}$pro-B/pre-B cells of mir-17-92 bKO mice (Supplementary Fig. 1b), suggesting that the almost intact expression of mir-17-92 allows for normal progression of early B lymphopoiesis in these mice. However, the expression of individual mir-17-92 member was greatly reduced $(>80 \%)$ in immature $\left(\mathrm{IgM}^{+} \mathrm{B}_{220}{ }^{+}\right)$and recirculating mature $\left(\mathrm{IgM}^{+}\right.$ $\mathrm{B} 220^{\text {high(hi) }}$ ) $\mathrm{B}$ cells in the BM of mir-17-92 bKO mice (Supplementary Fig. 1c,d) but these two B-cell populations were largely unperturbed (Fig. 1a).

The frequency of $\mathrm{B} 220^{+} \mathrm{IgM}^{+}$conventional B cells in the spleen and lymph nodes were also indistinguishable between mir-17-92 bKO and control mice (Fig. 1b). We further observed comparable percentage of follicular B $\left(\mathrm{B} 220^{+} \mathrm{CD} 93^{-} \mathrm{CD} 23^{+}\right.$ $\left.\mathrm{CD} 21^{-}\right)$, marginal zone $\mathrm{B}\left(\mathrm{B} 220^{+} \mathrm{CD} 93^{-} \mathrm{CD} 23^{-} \mathrm{CD} 21^{+}\right)$ and fraction I, II and III transitional B cells in the spleens of mir-17-92 bKO and control mice (Fig. 1c). B-cell follicles were maintained normally in mir-17-92 bKO mice (Fig. 1d). The mir-17-92 fl/fl alleles were indeed almost completely deleted (Supplementary Fig. 1e) and the expression of individual miRNAs significantly reduced $(>80 \%)$ in various mutant peripheral B-cell populations (Supplementary Fig. 1f-h). However, mir-17-92 bKO mice had slightly reduced $(\sim 20 \%)$ total B-cell numbers in their spleens and lymph nodes compared with control mice (Fig. 1e). As the enhanced expression of pro-apoptotic protein BIM, a known target of mir-17-92, was thought to account for the drastic reduction of mir-17-92-deficient pre-B cells ${ }^{18}$, we examined if the mild reduction of total peripheral B cells in mir-17-92 bKO mice could be ascribed to dysregulated BIM expression. Interestingly, Bim mRNA level was comparable between mir-17-92 bKO and control B cells (Fig. 1f), but BIM protein was enhanced in mir-17-92 bKO B cells (Fig. 1g,h), suggesting that mir-17-92 could modulate BIM expression at the protein level and the subtle decrease in total numbers of mutant peripheral B cells could be due to mildly increased BIM expression. Together, these results suggest that mir-17-92 cluster is not essential for conventional B-cell development in the periphery as it is for early B lymphopoiesis.

By contrast, both the frequency and total B-cell numbers were found to be reduced in the peritoneal cavities of mir-17-92 bKO mice compared with control mice (Fig. 2a,b). The reduction was ascribed to decreased $\mathrm{B} 1-\mathrm{a}\left(\mathrm{CD} 19^{+} \mathrm{CD} 43^{+} \mathrm{CD} 5^{+}\right)$and $\mathrm{B} 1-\mathrm{b}$ $\left(\mathrm{CD} 19^{+} \mathrm{CD} 43^{+} \mathrm{CD}^{-}\right)$cells as their numbers were reduced by approximately seven- and twofold, respectively, whereas conventional B (B2) $\left(\mathrm{CD} 19^{+} \mathrm{CD}^{-} 3^{-} \mathrm{CD}^{-}\right)$cells were not significantly affected. These data indicate that mir-17-92 is required for B1 cell development or maintenance.

Antibody responses in mir-17-92 bKO mice. We determined if mir-17-92 is required for B-cell function by examining antibody responses in mutant mice. When immunized with T-cellindependent (TI) antigen 4-hydroxy-3-nitrophenylacetyl (NP) 
coupled to Ficoll, which elicits IgM and IgG3 antibody production, mir-17-92 bKO mice exhibited significantly decreased NPspecific IgM and IgG3 antibody production compared with control mice at day 7 and 14 post immunization (Fig. 2c). This defect is consistent with the drastic reduction of B1 cells in these mice, which are the major responders to TI antigens ${ }^{23}$. Thus, mir17-92 is required for TI antibody response.

We next examined TD antibody response in mir-17-92 bKO mice by challenging them with NP coupled to chicken globulin (NP-CGG). We observed that mir-17-92 bKO and control mice produced similar amounts of NP-specific IgM and IgG1 antibodies, the two major antibody isotypes elicited by NPCGG immunization, at various time points post immunization (Fig. 3a). The $\mathrm{CD}_{19}{ }^{+} \mathrm{Fas}^{+} \mathrm{CD} 38^{-}$germinal centre (GC) B cells were comparably present in mir-17-92 bKO and control mice at day 10 post immunization, and class-switched antigen-specific $\mathrm{NIP}^{+}$IgG1 ${ }^{+}$(GC) B cells also appeared to be unperturbed in the mutant mice (Fig. 3b,c). We further stimulated $\mathrm{B}$ cells in vitro with anti-CD40 antibody and interleukin (IL)-4 and found IgG1 class switching to be indistinguishable between mutant and control B cells (Supplementary Fig. 2a). These data indicate that mir-17-92 is largely not required for GC response and the production of TD class-switched antigen-specific IgG1 antibodies.

Increased homing of PCs to BM in mir-17-92 bKO mice. We also examined in mutant mice the generation of memory $\mathrm{B}$ cells and long-lived PCs, which are differentiated through the GC response ${ }^{24,25}$. The frequency of NP-specific $\mathrm{CD} 38^{+} \mathrm{IgG} 1^{\text {high }}$ memory B cells was found to be similar in the spleen of mir-17-92 bKO and control mice at day 56 post immunization, suggesting that mir-17-92 is dispensable for memory B-cell generation and maintenance (Fig. 3d,e). However, when longlived PCs in the BM were examined by enzyme-linked immunospot (ELISPOT) assays, we consistently observed an increase $(20-30 \%)$ in the frequency of NP-specific IgG1 PCs in mir-17-92 bKO compared with control mice (Fig. 3f).

The increased long-lived PCs in the BM of mir-17-92 bKO mice prompted us to examine PCs in detail at early time points
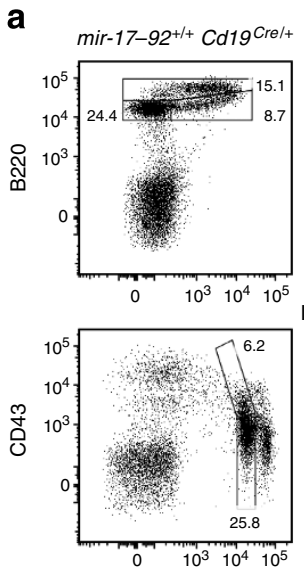

b
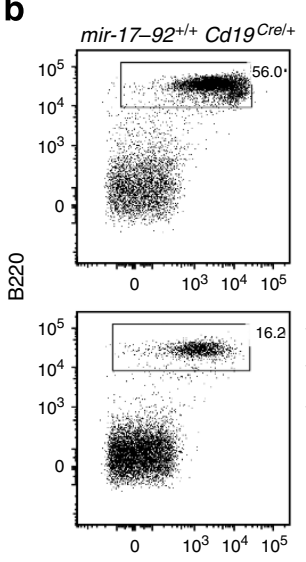
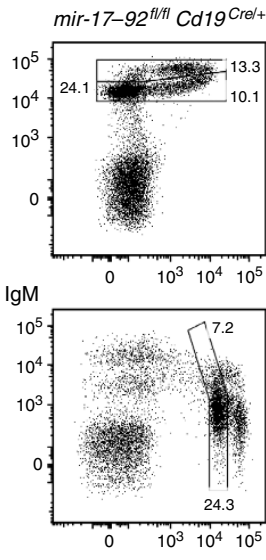

B220
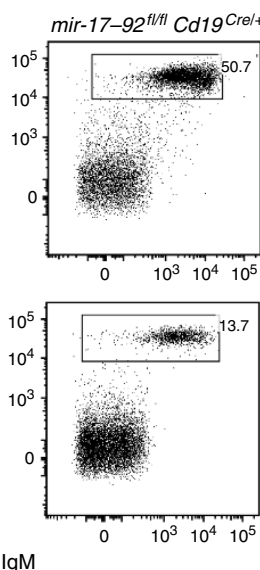

C
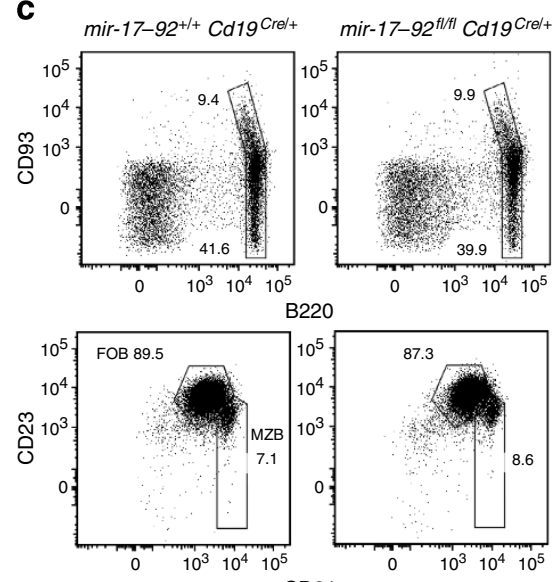

CD21

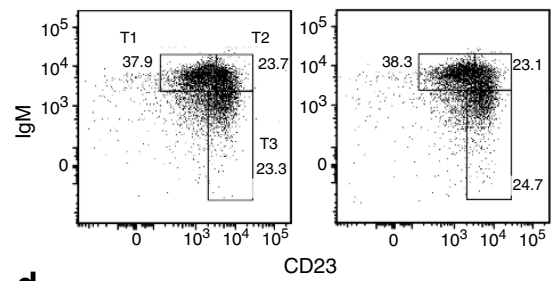

d

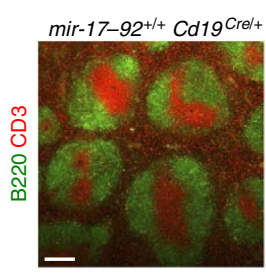

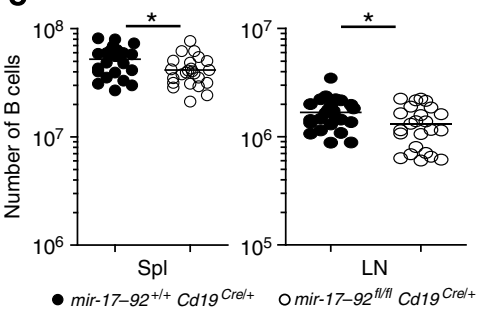

$\mathbf{f}$
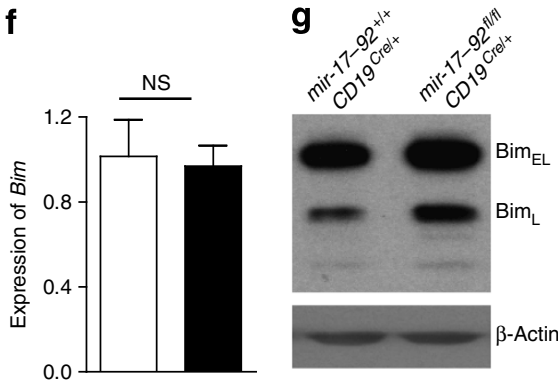

h

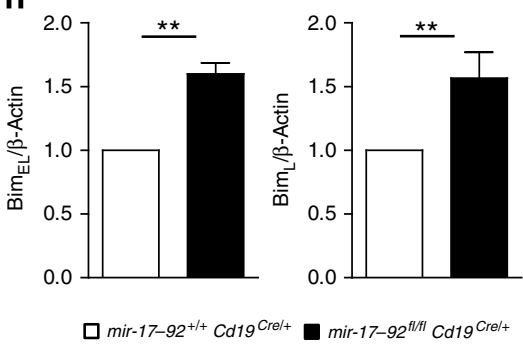

Figure 1 | Analyses of conventional B cells in mir-17-92 bKO mice. (a-c) FACS analyses of B cells in BM (a), spleen (b, upper panel and c) and inguinal lymph nodes (b, lower panel). B220 ${ }^{+} \mathrm{CD}_{3} 3^{-}$splenic B cells were separated into follicular B (FOB: $\left.\mathrm{CD} 23^{+} \mathrm{CD} 21^{-}\right)$and marginal zone B $(\mathrm{MZB}$ :

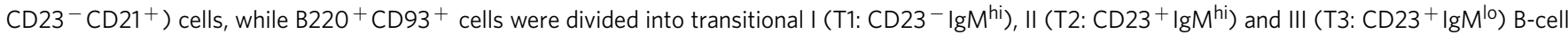
populations (c). Numbers indicate per cent cells in each gate. (d) Histology of spleens from unchallenged mir-17-92 control and bKO mice. B-cell follicles and T-cell areas were identified by B220 (green) and CD3 (red) staining. Scale bar, $100 \mu \mathrm{m}$. (e) Enumeration of IgM ${ }^{+} \mathrm{B}^{2} 20^{+} \mathrm{B}$ cells in spleen and inguinal lymph nodes. Cell numbers were calculated based on total cell count and their percentage as determined by FACS. (f) Expression of Bim mRNA in mir-17-92 bKO B cells. Splenic $\operatorname{lgM}{ }^{+} \mathrm{B}_{22}{ }^{+}$B cells from mir-17-92 control and bKO mice were examined for Bim mRNA expression by qRT-PCR. Bim expression in mir-17-92 bKO B cells was presented relative to that in control B cells. (g) BIM protein expression in mir-17-92 bKO B cells. Splenic lgM+B220 ${ }^{+}$B cells were purified and the cell lysates were subjected to western blotting with anti-BIM antibody. The antibody to BIM recognizes two isoforms: BIM extra long $\left(\right.$ Bim $\left._{E L}\right)$ and BIM long $\left(B_{L}\right)$. (h) Ratio of BIM to $\beta$-actin in mir-17-92 control and bKO B cells. The ratio in mir-17-92 control B cells was set as 1. Each symbol represents one mouse and horizontal lines indicate the mean. Unpaired two-tailed Student's $t$-test was used for statistical analyses. NS, not significant; ${ }^{\star} P<0.05 ;{ }^{\star} P<0.01$. Data shown are representative of more than five experiments $(\mathbf{a}, n=15 ; \mathbf{b}, n=24 ; \mathbf{c}, n=12 ; \mathbf{d}, \mathbf{f}$ and $\mathbf{g}, n=5)$. 
after immunization. Strikingly, at day 10 post immunization, the frequency of PCs was $\sim 2.5$-fold higher in the BM of mutant mice compared with controls (Fig. 4a,b). However, the frequency of splenic PCs from the same mice was not higher but rather reduced (Fig. 4a,b). The increased presence of PCs in the BM and decreased frequency of PCs in the spleen of mir-17-92 bKO mice was evident as early as day 7 post immunization (Fig. 4c). These results suggest that the increased PCs in the BM of immunized mir-17-92 bKO mice is not due to exaggerated generation of PCs in the spleen but could be due to the enhanced homing of splenic PCs to the BM during immune response. We further assessed if PC differentiation was perturbed in the absence of mir-17-92 by culturing B cells with lipopolysaccharide (LPS) in vitro. After 3 days of LPS stimulation, the percentage of $\mathrm{B} 220^{\text {low }} \mathrm{CD} 138^{+}$plasmablasts from mir-17-92 bKO and control B-cell cultures was comparable (Supplementary Fig. 2b), implying that mir-17-92 deficiency does not affect PC differentiation per se. Taken together, our data suggest that during an acute antibody response, $\mathrm{PC}$ differentiation is normal but homing to the $\mathrm{BM}$ is enhanced in the absence of mir-17-92.

Mir-17-92 directly targets S1pr1 expression in B cells. The homing of PCs from secondary lymphoid organs to BM is mainly mediated by sphingosine 1-phosphate (S1P) and chemokine CXCL12 through their engagements of S1PR1 (ref. 19) and chemokine-receptor CXCR4 (ref. 26), respectively. To understand how mir-17-92 modulates BM homing of PCs, we searched for possible targets of mir-17-92 using two miRNA target prediction software-TargetScan and MicroCosm, focusing on S1P-S1PR1 and CXCL12-CXCR4 signalling axes, and revealed that S1pr1 is a predicted target gene of mir-17-92 with five out of six members of the cluster (namely mir-17, mir-19a, mir-19b, mir-20a and mir-92a) targeting it.

We next determined if S1pr1 was indeed targeted by mir-1792. The $3^{\prime} \mathrm{UTR}$ of $S 1$ pr1 contains four predicted binding sites with mir-19a and mir-19b targeting two sites and mir-17, mir-20a and mir-92a each targeting one site (Fig. 5a). A luciferase reporter construct with full-length S1pr1 $3^{\prime}$ UTR DNA fragment was cloned downstream of firefly luciferase coding sequence and transfected into HEK293 cells together with either the whole mir-17-92 cluster or individual relevant miRNAs. We observed significantly reduced luciferase activity with the overexpression of either the whole cluster or individual miRNAs (Fig. 5b). We also generated reporter constructs containing individual wild-type (A to D) or mutant (Am to Dm)-binding sites where the DNA sequences complementary to the miRNA seed regions were mutated (Supplementary Fig. 3). When transfected into HEK293 cells with individual miRNAs, the constructs containing wild-type $3^{\prime}$ UTR yielded lower luciferase activities compared with the various mutant counterparts (Fig. 5c). Thus, our results suggest that S1pr1 is a bona fide target of mir-17-92, with five out of six members directly targeting its $3^{\prime}$ UTR at four different sites.

Modulation of S1PR1 expression in mir-17-92-deficient B cells. We proceeded to examine the effect of mir-17-92 deficiency on S1PR1 expression in B cells. First, splenic B220 ${ }^{+} \mathrm{IgM}^{+} \mathrm{B}$ cells were sorted and S1pr1 mRNA was examined by qRT-PCR. We found increased ( 30\%) S1pr1 mRNA level in mir-17-92 bKO B cells compared with controls (Fig. 5d), which was correlated with a slight increase in cell surface S1PR1 expression as determined by FACS analyses (Fig. 5e). We also examined S1pr1 mRNA in sorted $\mathrm{CD} 138^{+} \mathrm{B} 220^{\mathrm{low}}$ plasmablasts differentiated in vitro using LPS and $\mathrm{B}_{22}{ }^{-} \mathrm{CD} 138^{+} \mathrm{NIP}^{+}$plasmablasts from the spleens of immunized mice. We found that mir-17-92 deficiency led to increase in S1pr1 mRNA (Fig. 5f,h), which was corroborated by increased S1PR1 surface levels in the mutant cells (Fig. 5g,i). Hence, our data suggest that mir-17-92 modulates S1PR1 expression in PCs.

Next we investigated if modulating S1PR1 activity or expression in mir-17-92-deficient PCs could influence their homing to $\mathrm{BM}$. It is difficult to test S1P1 function in vitro due to receptor desensitization caused by non-physiological ligand exposure and the inability to model S1P concentration differential that exists across endothelial cells in vivo ${ }^{27}$. Thus, we examined BM homing
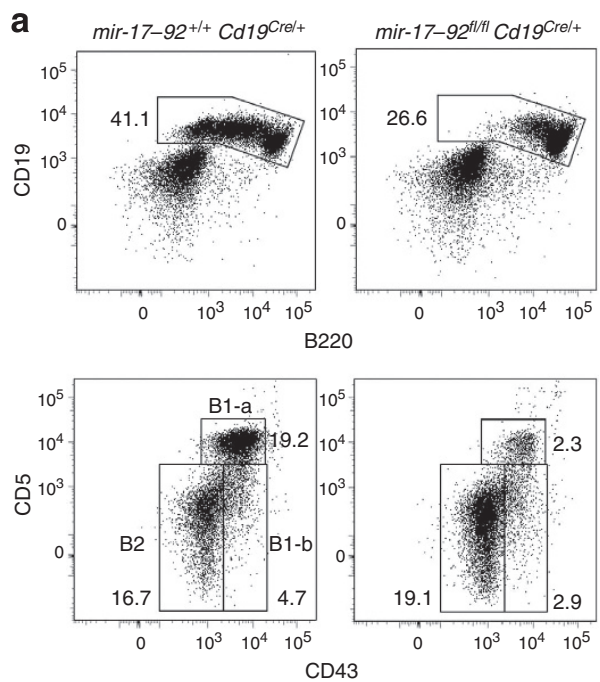
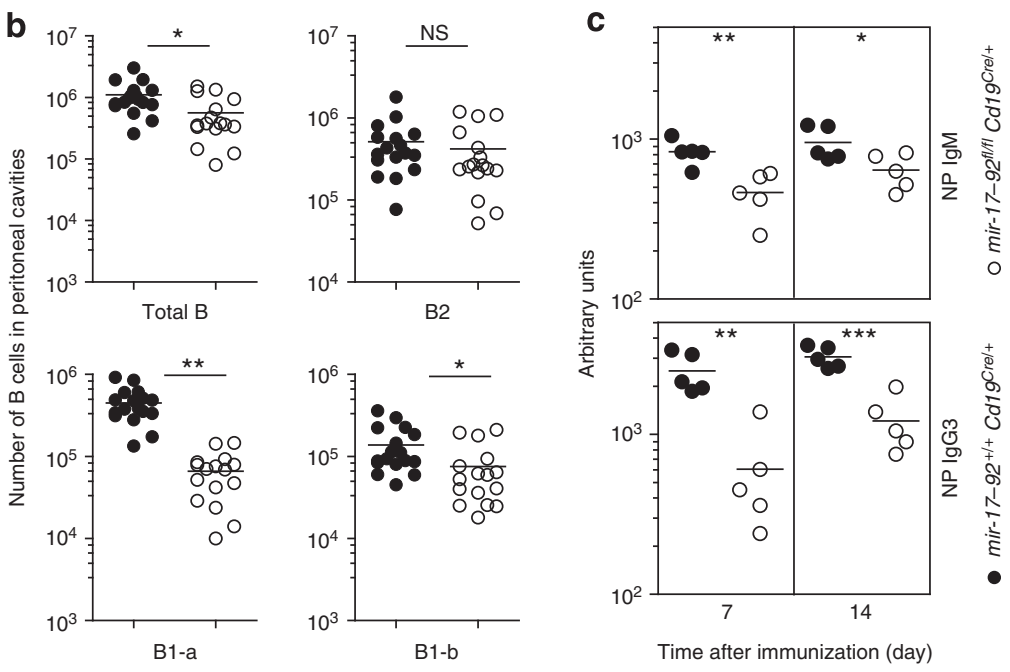

Figure 2 | Reduced B1 cells and defective TI antibody response in mir-17-92 bKO mice. (a) FACS analyses of peritoneal cavity B cells. CD19 ${ }^{+}$B22 ${ }^{+}$ $B$ cells were separated into conventional B2 $\left(C D 43^{-} \mathrm{CD} 5^{-}\right), \mathrm{B} 1-\mathrm{a}\left(\mathrm{CD} 43^{+} \mathrm{CD} 5^{+}\right)$and B1-b $\left(\mathrm{CD} 43^{+} \mathrm{CD} 5^{-}\right)$cells. Numbers indicate per cent cells in each gate. (b) Enumeration of various B-cell populations in the peritoneal cavities of mir-17-92 control and bKO mice. (c) Examination of NP-specific lgM and IgG3 antibody in mir-17-92 bKO mice. Mice were challenged with $\mathrm{TI}$ antigen $\mathrm{NP}_{17}$-Ficoll and sera were collected from immunized mice at day 7 and 14 post immunization. Serially diluted serum samples were subjected to ELISA for the detection of NP-specific IgM and lgG3 antibodies. Cell numbers were calculated based on the total cell count and their percentage as determined by FACS. Unpaired two-tailed Student s t-test was used for statistical analyses. NS, not significant; ${ }^{\star} P<0.05$; ${ }^{\star \star} P<0.01,{ }^{\star \star \star} P<0.001$. Each symbol represents one mouse and horizontal lines indicate the mean. Data shown are representative of 16 (a) and 2 (c) experiments. 
a

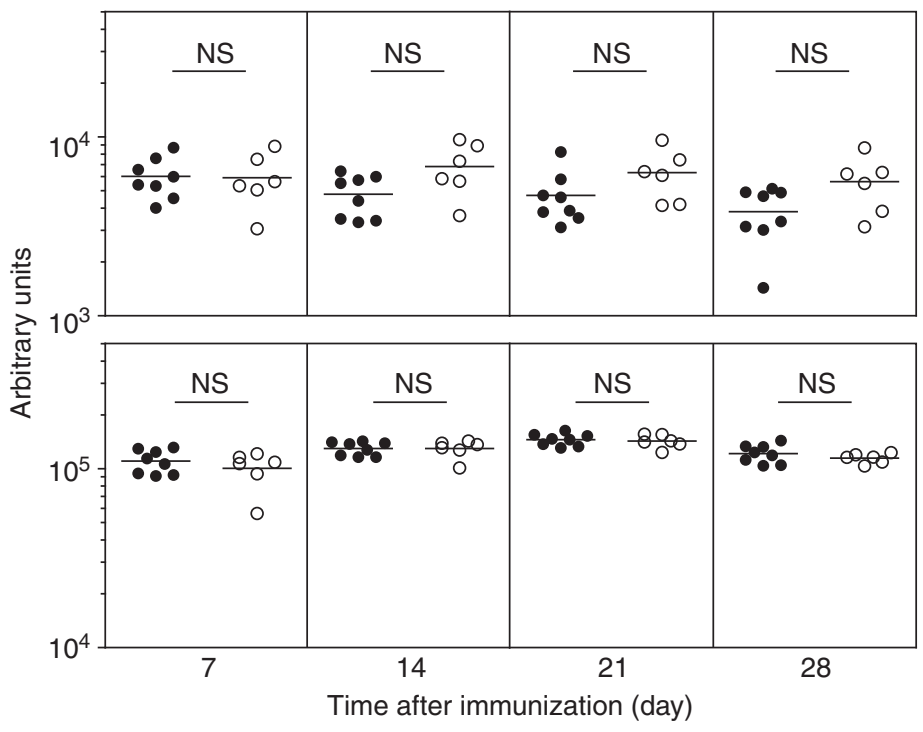

b $\quad$ mir-17-92+/+ Cd19Cre/+
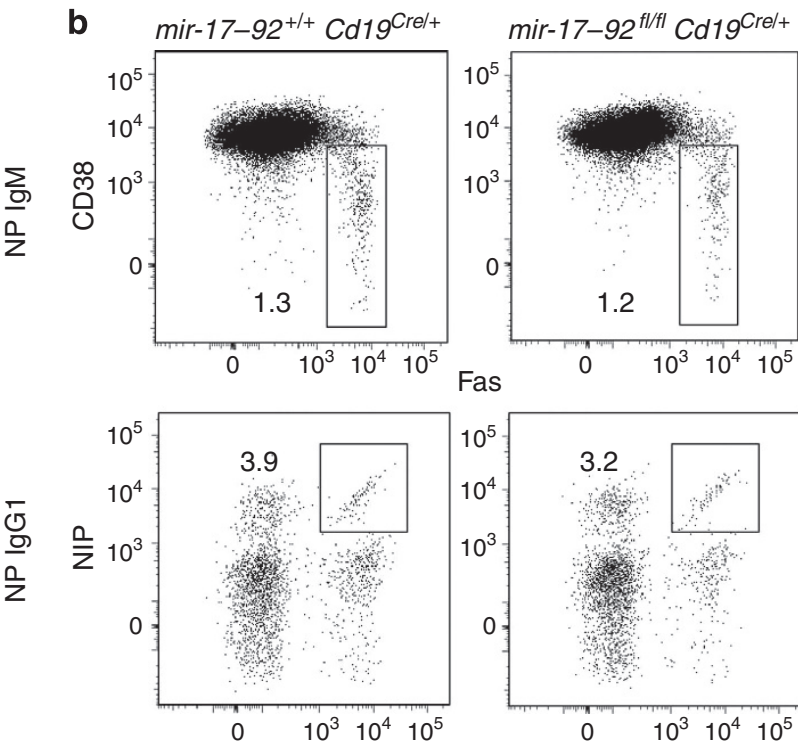

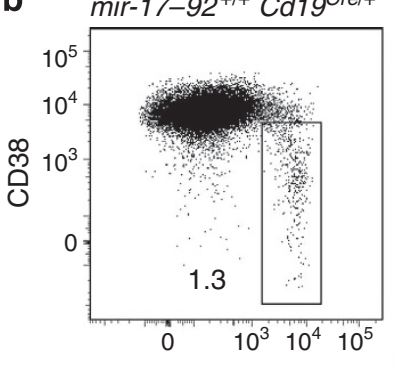

Fas

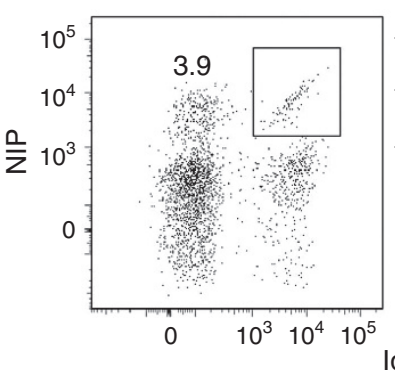

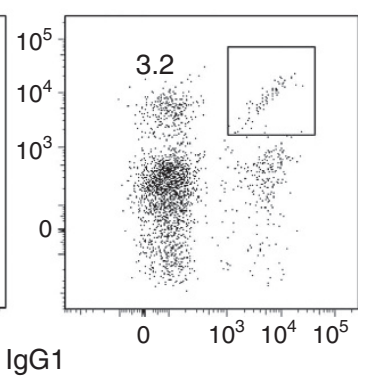

C
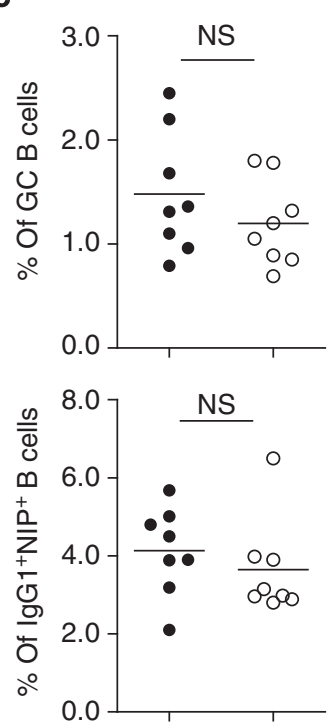

d
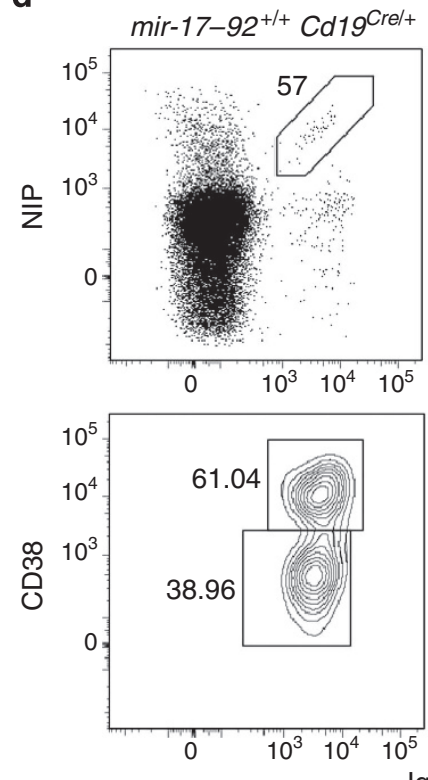
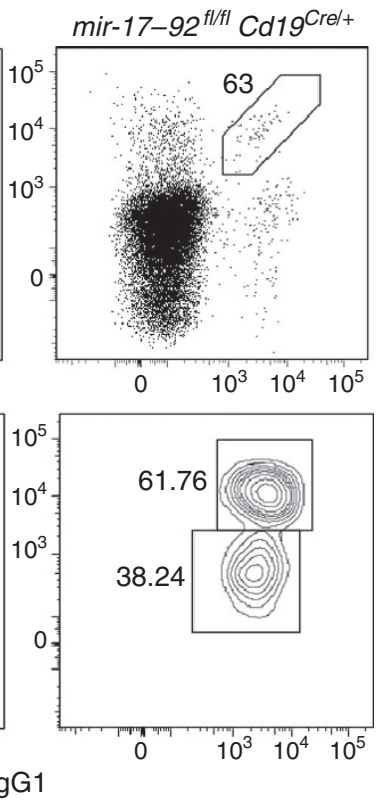

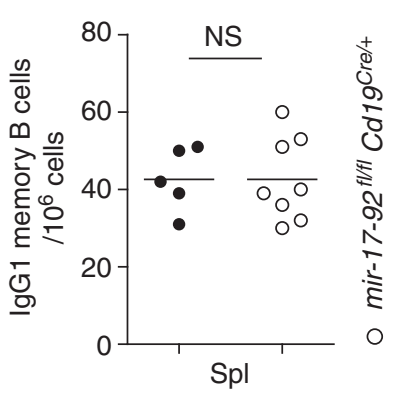

$\mathbf{f}$

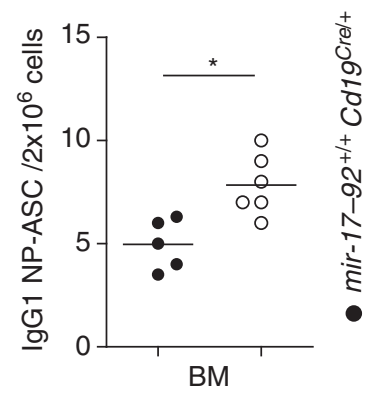

Figure 3 | TD antibody response in mir-17-92 bKO mice. (a) Examination of NP-specific IgM and IgG1 antibody in mir-17-92 bKO mice. Mice were challenged with TD antigen $\mathrm{NP}_{38}-\mathrm{CGG}$ in alum and the sera were collected from immunized mice at the indicated time points. Serially diluted serum samples were subjected to ELISA for the detection of NP-specific IgM and IgG1 antibodies. (b) FACS analysis of GC response in mir-17-92 bKO mice at day 10 post immunization. $\mathrm{CD}_{19}{ }^{+}$cells were stained for Fas ${ }^{+} \mathrm{CD}_{3} 8^{-} \mathrm{GC}$ B cells (upper panel) and $\mathrm{B} 22 \mathrm{O}^{+}$Dump ${ }^{-}$cells were stained for NP-specific NIPbinding IgG1 B cells (lower panel) from spleens. Numbers indicate per cent cells in each gate. (c) Frequency of GC and NIP ${ }^{+} \mid g G 1^{+} B$ cells in total and

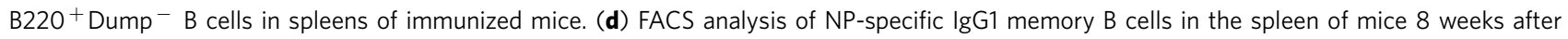
immunization. Splenic cells were stained for B220, IgG1 and CD38 expression and NIP binding after exclusion of dead cells and Dump cells; numbers adjacent to outlined areas indicate the number of cells within the gate among $1 \times 10^{6}$ total spleen cells (upper) or per cent cells in each gate (lower). (e) Frequency of NP-specific $\mathrm{CD}_{3} 8^{+} \mathrm{lgG} \mathrm{h}^{\text {high }}$ memory B cells in spleens of mir-17-92 control and bKO mice as analysed in d. (f) Frequency of long-lived PCs in the BM of mir-17-92 control and bKO mice. NP-specific lgG1 antibody-secreting cells (ASCs) in the BM of mir-17-92 control and bKO mice were determined by ELISPOT assays 8 weeks after immunization and shown as numbers of ASCs per $2 \times 10^{6}$ BM cells. Unpaired two-tailed Student's $t$-test was used for statistical analyses. NS, not significant; ${ }^{\star} P<0.05$. Each symbol represents one mouse and horizontal lines indicate the mean $(\mathbf{a}, \mathbf{c}, \mathbf{e}, \mathbf{f})$. Data shown are representative of two (a, $n=6$ to 8$)$, six (b) or eight (d) experiments.

of PCs in immunized mir-17-92 bKO mice in response to different dosages of FTY720 (Fig. 6a), a potent S1P1 inhibitor ${ }^{28,29}$ that impedes the BM homing of PCs from secondary lymphoid tissues $^{19}$. We reasoned that higher S1PR1 expression level in mir17-92-deficient PCs would render them less sensitive to FTY720 inhibition. Consistent with previous report ${ }^{19}$, when mice were given high dose of FTY720 (3.0 $\left.\mathrm{mg} \mathrm{kg}^{-1}\right)$, there was hardly any PCs detected in the BM of both mir-17-92 control and bKO mice at day 10 post immunization (Fig. 6b). Interestingly, when FTY720 dosage was reduced to $1.0 \mathrm{mg} \mathrm{kg}^{-1}$, NP-specific PCs were found in the BM of mir-17-92 bKO but not control mice. When the dosage was further lowered to $0.3 \mathrm{mg} \mathrm{kg}^{-1}$, we could 


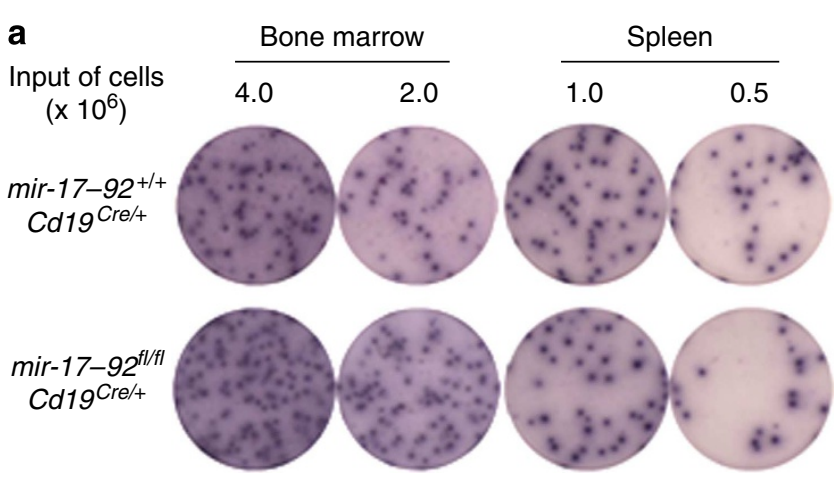

b
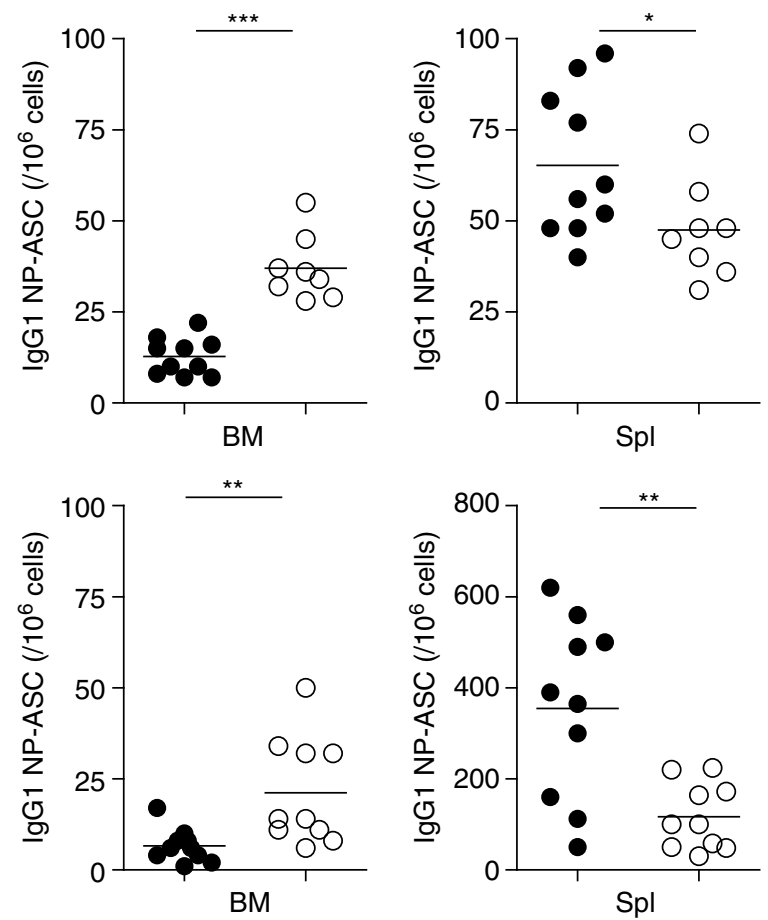

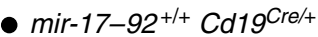

O mir-17-92 ${ }^{\mathrm{fl} / \mathrm{fl}} \mathrm{Cd} 19^{\mathrm{Cre} / \mathrm{+}}$

Figure 4 | Enhanced BM homing of PCs in mir-17-92 bKO mice.

$(\mathbf{a}, \mathbf{b})$ NP-specific IgG1 ASCs in the BM and spleens of mir-17-92 control and bKO mice at day 10 post immunization with $\mathrm{NP}_{38}$-CGG. ASCs in $\mathrm{BM}$ and spleens were examined by ELISPOT assays, shown as images of assays (a) and frequency of NP-specific IgG1 ASCs (b). (c) Frequency of NP-specific lgG1 ASCs in the BM and spleens of mir-17-92 control and bKO mice at day 7 after immunization. Unpaired two-tailed Student's $t$-test was used for statistical analyses. ${ }^{\star} P<0.05 ;{ }^{* \star} P<0.01 ;{ }^{* \star} P<0.001$. Each symbol represents one mouse and horizontal lines indicate the mean $(\mathbf{b}, \mathbf{c})$. Data shown are representative of eight (a) experiments.

detect NP-specific PCs in the BM of both mir-17-92 control and bKO mice but with the frequency of PCs significantly higher (approximately threefold) in the latter (Fig. 6b). These data are consistent with our hypothesis that a higher level of S1PR1 expression in mir-17-92-deficient PCs renders them less sensitive to FTY720 inhibition.

To further determine if increased S1PR1 expression indeed contributed to enhanced PCs homing to the BM in mir-17-92 bKO mice, we generated mir-17-92 flfl S1pr fll $+C d 19^{\mathrm{Cre} /+}$ mice that have one S1pr1 allele specifically deleted in B cells. We reasoned that $S 1$ pr1 haploinsufficiency could reduce the elevated expression caused by mir-17-92 deficiency and therefore normalize the PC homing phenotype in these mice. First, we examined
S1pr1 expression in mir-17-92 $2^{f l f l} S 1 p r 1^{+/+} C d 19^{C r e /+}$ and mir-17-92 fl/fl S1pr1 fll $+C d 19^{C r e l}+\mathrm{B}$ cells and found that S1pr1 was reduced $\sim 45 \%$ when one S1pr1 allele was ablated (Fig. $6 \mathrm{c}$ ). We next immunized the mice with NP-CGG and examined NP-specific PCs in the BM at day 10 post immunization by ELISPOT assay. As expected, the number of NP-specific IgG1 PCs in the BM of mir-17-92 fl/fl S1pr $1^{+/+} \mathrm{Cd} 19^{\mathrm{Cre} /+}$ mice was increased compared with mir-17-92 $+/+S 1$ pr ${ }^{+/+} \mathrm{Cd} 19^{\mathrm{Cre} /+}$ mice (Fig. 6d,e). Interestingly, when one S1pr1 allele was ablated, the number of NP-specific IgG1 PCs in the BM of mir-17-92 fl/fl $S 1$ pr $f^{f l}+C d 19^{C r e l}+$ mice was reduced compared with mir-17-92 fl/fl S1pr1 ${ }^{+1+} \mathrm{Cd} 19^{\mathrm{Cre} /+}$ mice and became similar to that found in mir-17-92 $+/+S 1 p r 1^{+/+} C d 19^{C r e /+}$ mice. These results indicate that increased S1PR1 expression indeed contributes to the increased BM homing of PCs in mir-17-92 bKO mice. Taken together, our data suggest that mir-17-92 restrains BM homing of PCs by directly suppressing S1PR1 expression.

Impaired IgG2c antibody production in mir-17-92 bKO mice. During our initial characterization of mir-17 92 bKO mice, we found normal levels of IgM, IgG1, IgG3 and IgG2b, but significantly reduced IgG2c antibodies, in the sera of unchallenged mutant mice compared with controls (Fig. 7a). It is known that inbred mouse strains such as C57BL/6 and SJL with the Igh-1b allele possess only the gene encoding the IgG2c isotype, whereas $\mathrm{BALB} / \mathrm{c}$ and $129 / \mathrm{Sv}$ strains with the Igh-1a allele express only IgG2a (ref. 30). The allele-specific PCR analysis confirmed the presence of IgG2c but not IgG2a gene alleles in mice used in our study (Supplementary Fig. 4), which have been backcrossed to C57BL/ 6 background for more than 10 generations, and therefore ruled out the possibility that the reduced serum IgG2c in mutant mice was due to different genetic backgrounds.

We next examined if the production of antigen-specific IgG2c antibodies would be affected during acute antibody response. Interestingly, mir-17-92 bKO mice had markedly reduced $\mathrm{NP}$-specific IgG2c antibodies at various time points post immunization compared with control mice (Fig. 7b). This is in contrast to the normal production of NP-specific IgG1 antibodies in mir-17-92 bKO mice (Fig. 3a). To exclude the possibility that defective IgG2c production in mir-17-92 bKO mice could be due to the modest reduction of peripheral B-cell numbers in these mice, we generated and analysed mir-17-92 fl/fl AicdaCrel + mice in which the floxed mir-17-92 allele was deleted only in antigenactivated but not naïve B cells (Supplementary Fig. 5a). Unchallenged mir-17-92 ${ }^{f l f l}$ AicdaCre/+ mice had normal B-cell populations in their spleens and lymph nodes compared with mir-17-92 ${ }^{+/+}$AicdaCre/+ mice (Supplementary Fig. 5b,c). NP-specific IgG1 antibodies were also not drastically affected in mir-17-92 ${ }^{f l f l}$ Aicda Crel+ mice, especially at later time points post immunization (Supplementary Fig. 5d). In contrast, the production of NP-specific IgG2c antibodies was significantly reduced in mir-17-92 $2^{f l f t}$ AicdaCrel + mice (Fig. 7c), suggesting that mir-17-92 is indeed important for IgG2c antibody production.

To further determine if mir-17-92-deficient B cells were intrinsically defective in IgG2c production, we purified B cells from mir-17-92 bKO and control mice and stimulated them in vitro with LPS + IFN- $\gamma$, which induces preferential Ig class switching to IgG2c/2a, and assessed the presence of IgG2 $\mathrm{c}^{+}$cells by FACS analysis. After 3 days, mir-17-92 bKO B-cell culture generated significantly reduced IgG $2 \mathrm{c}^{+}$cells $(\sim 30 \%$ of control; Fig. $7 \mathrm{~d}, \mathrm{e})$. However, the generation of $\mathrm{IgG}^{+}$and $\operatorname{IgG}^{+} \mathrm{B}$ cells after LPS and LPS + IL-4 stimulations, respectively, was comparable between mir-17-92 bKO and control cultures. The switching to IgG2b in response to LPS + TGF $\beta+$ IL- 5 stimulation appeared to be reduced but it was not statistically significant (Fig. 7d,e). 
a

S1pr1 3'-UTR $(1.3 \mathrm{~kb})$

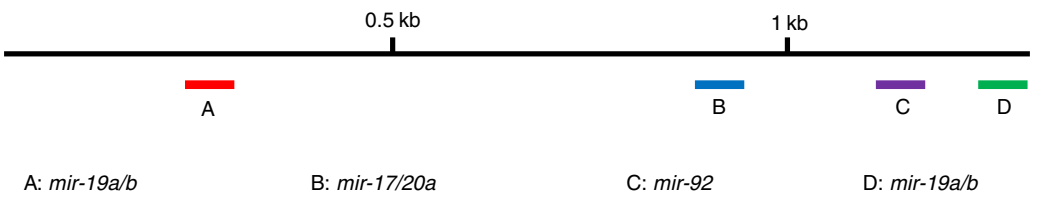

b

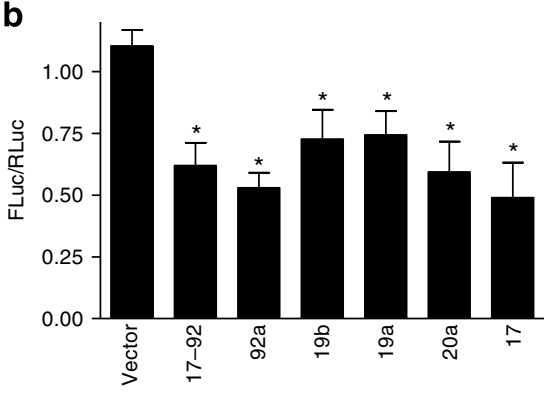

C
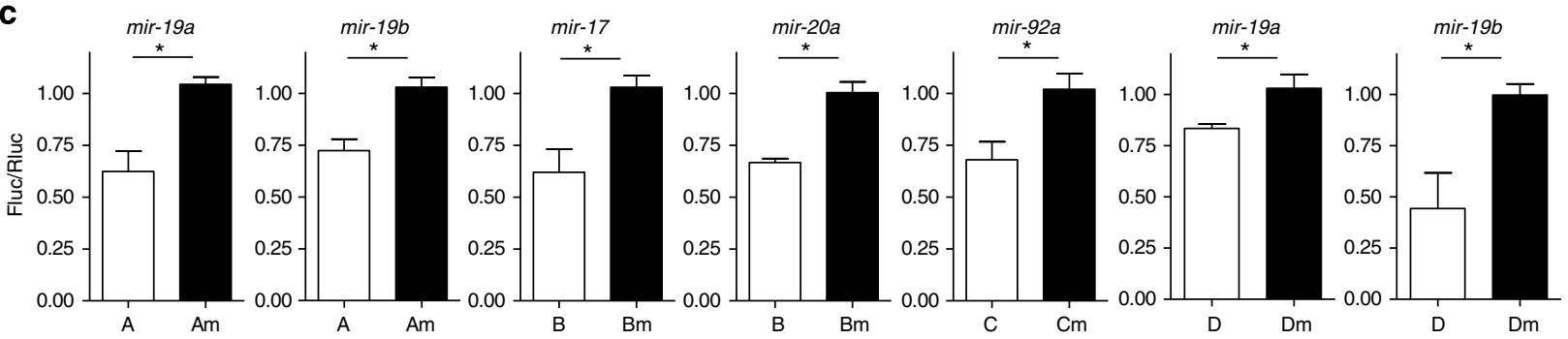

d

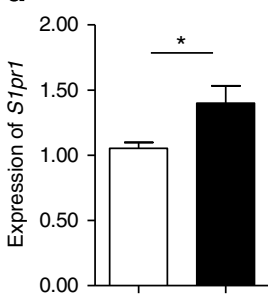

e

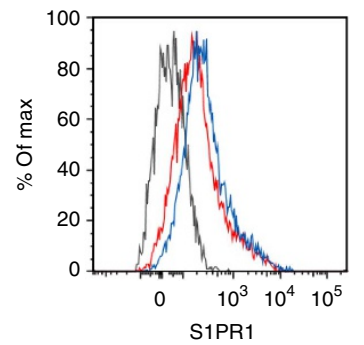

h

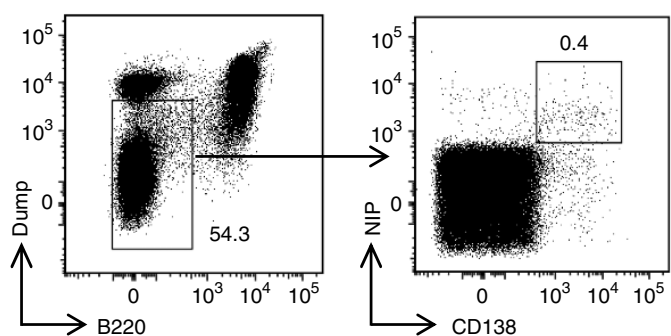

f

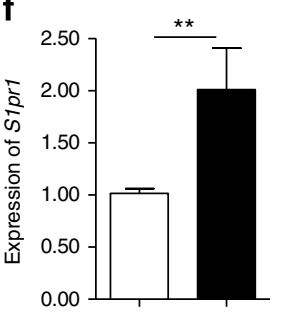

g

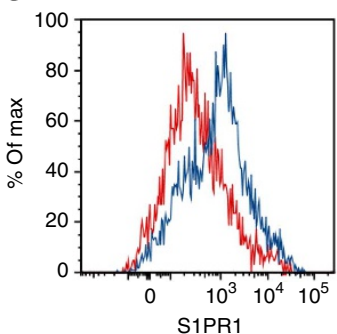

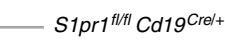

- mir-17-92+/+ Cd19Crel+ mir-17-92 ${ }^{\mathrm{fl} / \mathrm{fl}} \mathrm{Cd} 1 \mathrm{~g}^{\mathrm{Cre} / \mathrm{+}}$

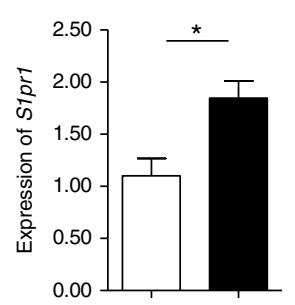

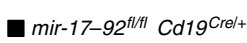

$\square$ mir-17-92+/+ Cd19Crel+

Figure 5 | Mir-17-92 targets S1pr1 in PCs. (a) Schematic representation of S1p1 3'UTR and the four predicted binding sites (A to D) for mir-17-92. $(\mathbf{b}, \mathbf{c})$ Luciferase reporter assay validating mir-17-92 targeting of S1pr1. HEK293 cells were transfected with full-length S1pr1 3'UTR construct and vectors containing mir-17-92 cluster or individual mir-17-92 members or control vector (b), or with various WT (a-d) or mutant (Am to Dm) S1p1 3'UTR constructs and individual miRNAs as depicted (c). Firefly luciferase activity is normalized to renilla luciferase activity (Fluc/Rluc). Results are presented relative to control, set as 1. The Fluc/Rluc for WT 3'UTR constructs (a-d) was presented relative to that for corresponding mutant constructs (Am to Dm).

(d,f,h) qRT-PCR analyses of S1pr1 mRNA in sorted resting B220 ${ }^{+} \mathrm{IgM}{ }^{+}$B cells (d), LPS-induced CD138 ${ }^{+}$B220 low (f) and antigen-specific

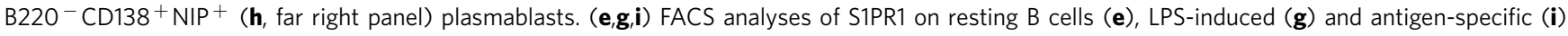
plasmablasts. Mice were immunized twice with $\mathrm{NP}_{38}-\mathrm{CGG}$ at day 0 and day 28 and sacrificed at day 32 . $\mathrm{NIP}^{+} \mathrm{CD}^{-} 8^{+}$plasmablasts were sorted from Dump ${ }^{-}{\text {B } 220^{-}}^{-}$spleen cells (h, left and middle panels). Resting B cells from S1pr $7^{f / f l} \mathrm{Cd} 19^{\mathrm{Cre} /}+$ mice were served as a negative control (grey) for S1PR1 staining. Bar graphs represent mean \pm s.d. $(n=3)$. Expression of S1pr1 in mir-17-92 bKO cells (white) was presented relative to that in control cells (black). Unpaired two-tailed Student's $t$-test was used for statistical analyses. ${ }^{\star} P<0.05 ;{ }^{\star \star} P<0.01$. Data are representative of three (b-e,g,i) or five (f) experiments.

These data suggest that mir-17-92 is intrinsically required for IgG2c production in B cells.

Mir-17-92 regulates GLT and histone acetylation at $\mathrm{C} \gamma 2 \mathrm{c}$ locus. We explored how mir-17-92 could regulate IgG2c production. As AID is absolutely required for Ig class switch recombination (CSR), we first assessed if the gene expression of Aicda was affected in mir-17-92-deficent B cells using qRT-PCR. We found comparable Aicda expression between stimulated mir-17-92 bKO and control B cells (Supplementary Fig. 6), thus excluding the possibility that the defective $\operatorname{IgG} 2 \mathrm{c}$ production was due to dysregulated AID expression. Next we examined germline transcription (GLT) in IgH switch (S) regions, which is important for AID to access ssDNA targets ${ }^{31}$. When germline transcripts for $\gamma 3$, $\gamma 1, \gamma 2 \mathrm{c}$ and $\gamma 2 \mathrm{~b}$ in various B-cell cultures were determined by $\mathrm{qRT}-\mathrm{PCR}$, we detected marked reduction in $\gamma 2 \mathrm{c}$ GLT in mir-17-92 bKO cells stimulated with LPS + IFN $\gamma$ compared with controls, whereas $\gamma 3, \gamma 1$ and $\gamma 2 \mathrm{~b}$ GLT levels were largely comparable between mutant and control cells (Fig. 7f).

Chromatin remodelling is required for many DNA-directed processes, such as transcription, recombination and replication. 
a

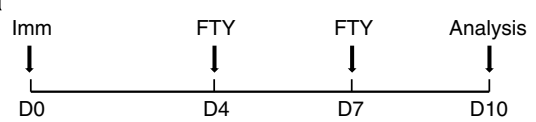

b

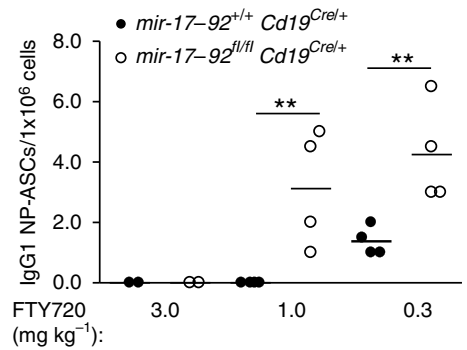

C

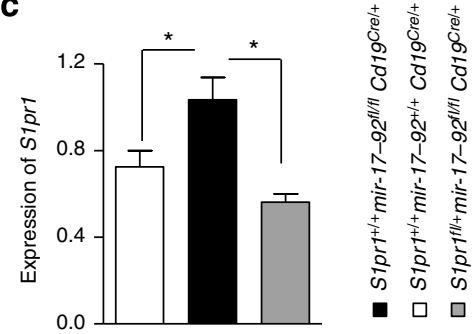

d

Input of cells $\left(x 10^{6}\right)$

2.0

1.0

$\begin{array}{cccc}\text { S1pr1 } & +/+ & +/+ & f l /+ \\ \text { mir-17-92 } & +/+ & f l / f l & f l / f l \\ \text { Cd19 } & \text { Cre/+ } & \text { Cre/+ } & \text { Cre/+ }\end{array}$

e

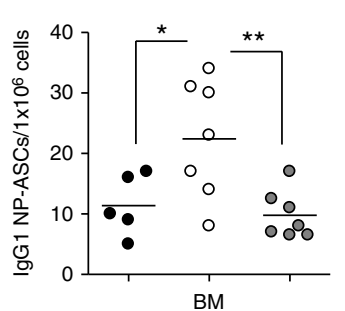

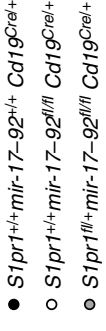

Figure 6 | Effects of FTY720 inhibition and S1pr1 haploinsufficiency on mir-17-92 bKO PCs. (a) Timeline of FTY720 treatment and immunization of mice. Mice were immunized with NP 38 -CGG at day 0 and given FTY720 at different dosages at day 4 and day 7 before analyses at day 10. (b) Frequency of NP-specific IgG1 ASCs in the BM of FTY720-treated mice at day 10 after immunization. (c) Reduced S1pr1 expression by S1pr1 haploinsufficiency. S1pr1

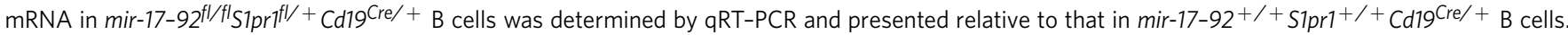

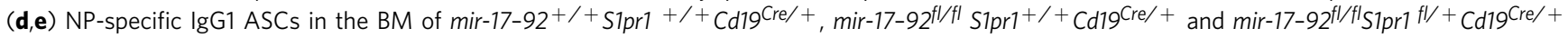
mice at day 10 post immunization. NP-specific IgG1 ASCs were determined by ELISPOT assays, shown as images of assays (d) and frequency (e). Unpaired two-tailed Student's $t$-test was used for statistical analyses. ${ }^{\star} P<0.05 .{ }^{\star \star} P<0.01$. Each symbol represents one mouse and small horizontal lines indicate the mean $(\mathbf{b}, \mathbf{e})$. Bar graphs represent mean \pm s.d. $(n=3)$. Data are representative of three $(\mathbf{c})$ or five $(\mathbf{d})$ experiments.

Acetylation (Ac) on amino-terminal tails of histone $\mathrm{H} 3$ and $\mathrm{H} 4$ at $\mathrm{S}$ regions has been shown to be important for Ig GLT and $\mathrm{CSR}^{32,33}$. We therefore performed chromatin immunoprecipitation (ChIP)-qPCR to assess the acetylation of histone $\mathrm{H} 3$ $(\mathrm{H} 3 \mathrm{Ac})$ and $\mathrm{H} 4(\mathrm{H} 4 \mathrm{Ac})$ at the $\mathrm{S}$ region and $\mathrm{C} \gamma 2 \mathrm{c}$ I exon of LPS + IFN $\gamma$-treated B cells using specific antibodies. When measured by ChIP-qPCR, mutant cells had lower H3Ac levels were lower at the $\mathrm{S}$ region and $\mathrm{C} \gamma 2 \mathrm{c}$ I exon compared with control cells at $48 \mathrm{~h}$ after LPS + IFN $\gamma$ stimulation (Fig. 8a), correlating with decreased IgG2c GLT in mutant B cells (Fig. 7f). Consistent with the normal IgG1 GLT (Fig. 7f), H3Ac levels at $S \gamma 1 / \mathrm{I} \gamma 1$ loci were comparable between mutant and control B cells stimulated with LPS + IL-4 (Fig. 8b). We also examined H4Ac at $\mathrm{C} \gamma 2 \mathrm{c} S$ region, which is known to reflect AID accessibility and activity at $S$ regions and important for $\mathrm{CSR}^{32}$. We showed that H4Ac level at S $\gamma 2 \mathrm{c}$ was lower in LPS + IFN $\gamma$-stimulated mir-1792 bKO cells compared with similarly treated control cells (Fig. 8c), indicating reduced AID accessibility at $S \gamma 2 c$ region of mutant cells. However, $\mathrm{H} 4 \mathrm{Ac}$ level at the $\mathrm{S} \gamma 1$ region was comparable in LPS + IL-4-treated mir-17-92 bKO and control cells (Fig. 8d). Taken together, these results suggest that mir-1792 deficiency affects chromatin remodelling at $\mathrm{C} \gamma 2 \mathrm{c} / 2 \mathrm{a}$ locus and consequently, GLT, AID accessibility and CSR.

Mir-92a directly targets $I k z f 1$ and regulates IgG2c production. As miRNAs negatively regulate gene expression, we hypothesized that mir-17-92 could target negative regulator(s) of IgG2c CSR that likely also regulates chromatin remodelling. Hence, the absence of mir-17-92 would lead to the derepression of negative regulator(s) and result in reduced $\operatorname{IgG} 2 \mathrm{c}$ production. It was previously shown that IKAROS deficiency led to increased and ectopic switching to IgG2c/2a (ref. 20). IKAROS was also showed to maintain repressive chromatin at $\mathrm{C} \gamma 2 \mathrm{c} / 2 \mathrm{a}$ and suppress GLT, AID accessibility and CSR of IgG2a/2c (ref. 20). More recently, $I k z f 1$, the gene encoding IKAROS, was demonstrated to be directly targeted by mir-92a in T cells ${ }^{21}$. Given that mir-17-92deficient B cells exhibited reduced GLT and hypoacetylation of histone at $\mathrm{S} \gamma 2 \mathrm{c}$ region, we postulated that mir-17-92 likely regulated IgG $2 \mathrm{a} / 2 \mathrm{c}$ switching by modulating IKAROS expression in activated B cells. We found that $I k z f 1$ was indeed targeted by mir-17-92 or mir-92a but not by other members of the cluster using luciferase reporter assays (Supplementary Fig. 7). We demonstrated that mir-17-92-deficient B cells had higher ( 60\%) Ikzf1 mRNA levels than control cells when treated with LPS + IFN $\gamma$ (Fig. 8d). Consistent with increased Ikzf1 mRNA, the protein level of IKAROS was also enhanced in stimulated mutant cells as determined by western blotting (Fig. 8e,f). These results together indicate that mir-17-92 directly targets $I k z f 1$ and modulates IKAROS expression in B cells.

We next asked if elevated IKAROS expression could account for the defective IgG2c production in mir-17-92-deficient B cells. We used short interfering RNA (siRNA) to knock-down IKAROS in the mir-17-92-deficient cells with nucleofection, which can efficiently transfect activated B cells in vitro (Supplementary Fig. 8). Western blotting analyses showed that IKAROS protein level was noticeably reduced in mutant B cells transfected with Ikzf1-targeting siRNA compared with control siRNA (Fig. 9a). More importantly, Ikzf1-silenced mutant B cells had increased IgG2c class switching compared with non-silenced counterparts 
a
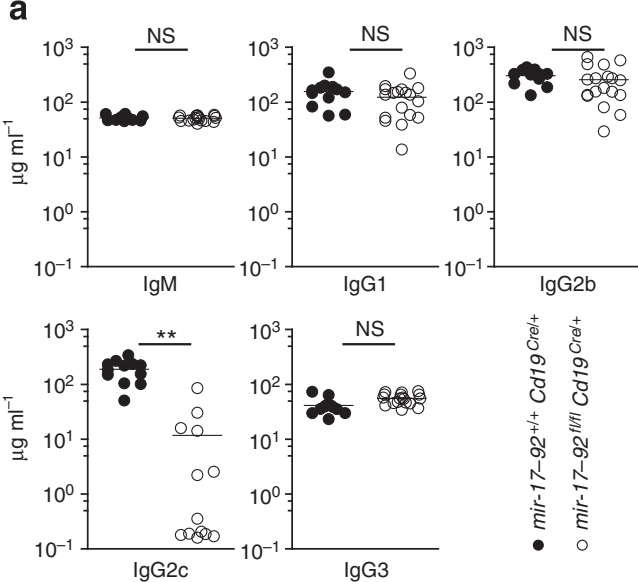

b
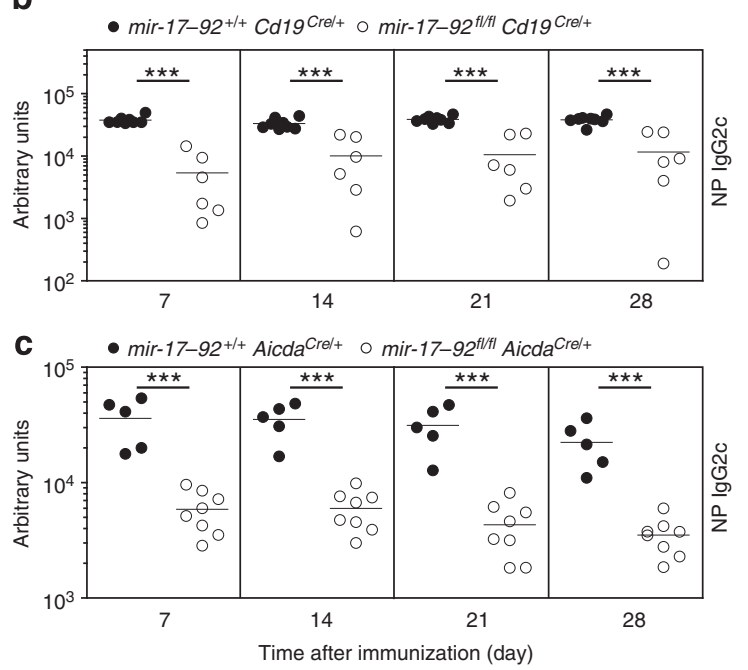

d
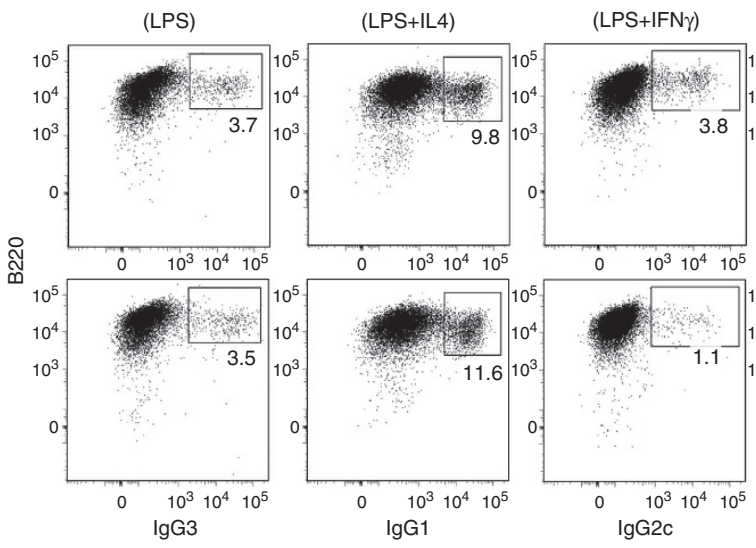

(LPS+TGF $\beta+$ IL5)

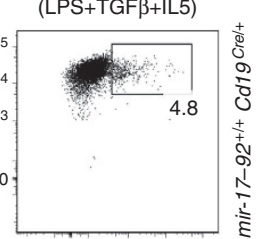

e
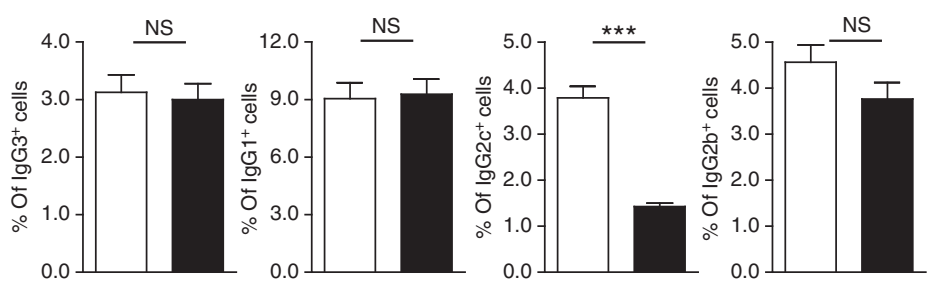

f
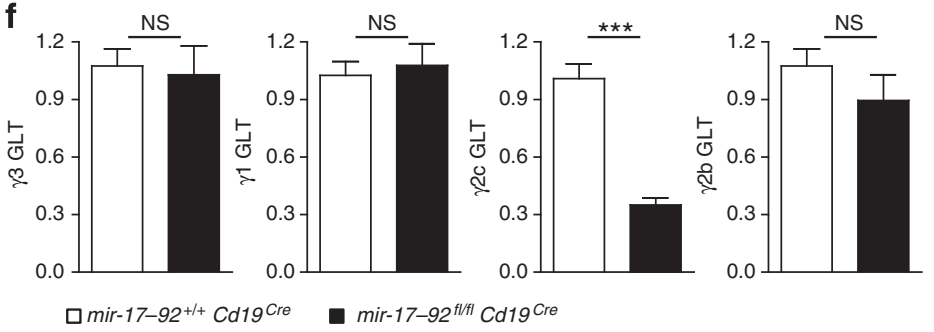

Figure 7 | MiR-17-92 is required for IgG2c production. (a-c) Reduced production of IgG2c antibodies in miR-17-92-deficient mice. For basal Ig measurement, sera were collected from unchallenged mir-17-92 control and bKO mice (a). Mir-17-92 control and bKO mice (b) or mir-17-92+/ +

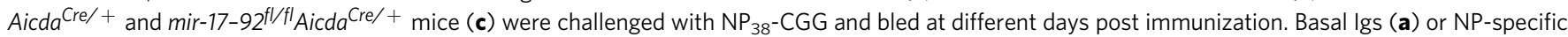
IgG2c antibodies (b,c) were examined by ELISA. (d) Defective IgG2c class switching of in vitro-stimulated mir-17-92 bKO B cells. B cells were cultured for 3 days with LPS, LPS + IL-4, LPS + IFN $\gamma$ and LPS + TGF $\beta+$ IL-5 and class-switched IgG3 ${ }^{+}, \operatorname{lgG} 1^{+}, \operatorname{lgG} 2 \mathrm{c}^{+}$and IgG2b ${ }^{+} \mathrm{B}$ cells were analysed by FACS analyses. (e) Percentage of $\operatorname{lgG} 3^{+}, \operatorname{lgG} 1^{+}, \operatorname{lgG} 2 \mathrm{c}^{+}$and $\operatorname{lgG} 2 \mathrm{~b}^{+} \mathrm{B}$ cells as cultured in vitro and analysed in d. (f) Reduced $\operatorname{lgG} 2 \mathrm{c}$ germline transcript (GLT) in the absence of mir-17-92. GLTs of $\gamma 3, \gamma 1, \gamma 2 c$ and $\gamma 2 b$ in mir-17-92 bKO B cells were determined by qRT-PCR and presented relative to that in control B cells. Unpaired two-tailed Student's $t$-test was used for statistical analyses. NS, not significant; ${ }^{\star \star} P<0.01,{ }^{\star \star \star} P<0.001$. Each symbol represents one mouse and horizontal lines indicate the mean $(\mathbf{a}-\mathbf{c})$. Bar graphs represent mean \pm s.d. $(\mathbf{e}, n=10 ; \mathbf{f}, n=5)$. Data shown are representative of 2 (a, $n=15$ to $18 ; \mathbf{b}$, $n=6$ to $8 ; \mathbf{c}, n=5$ to 8 ) and 10 (d) experiments.

(Fig. 9b,c). We further demonstrated that introduction of mir-92a mimics but not others could enhance IgG2c production in mir-17-92-deficient and control B cells (Fig. 9d). Taken together, our data suggest that mir-92a from mir-17-92 cluster directly represses IKAROS expression and regulates IgG2c production.

Mir-17-92 deficiency attenuates murine autoimmunity. IgG2c/ $2 \mathrm{a}$ is the most prevalent autoantibody isotype found in murine lupus $^{34,35}$. These autoantibodies bind self DNA and ribonucleoproteins to form immune complexes that cause tissue damage. The systemic autoimmunity in mir-17-92-transgenic mice $^{36,37}$ and selective IgG2c defect in mir-17-9fl/fl Cd19 Crel+ mice led us to explore if mir-17-92 ablation could attenuate B-cell-mediated autoimmunity. Hence, we introduced mir-17-92 deficiency into Ptpn $6^{f l f l} C d 19^{C r e /+}$ mice that had Shp1 tyrosine phosphatase deleted in $\mathrm{B}$ cells and developed autoimmunity resembling that of human lupus ${ }^{38}$.
Compared with age-matched Ptpn $6^{+/+}$mir-17-92 $+/+$ Cd19Crel + control mice, 10-week-old Ptpn $6^{f l / f l}$ mir-17-92 $+/+$ $\mathrm{Cd} 19^{\mathrm{Cre} /}+$ mice manifested increased sera titres of $\operatorname{IgM}$ and IgG2c antibodies reactive to single-stranded (ss) and doublestranded (ds) DNA. Interestingly, sera titres of anti-ssDNA and anti-dsDNA IgM antibody were diminished by $50-60 \%$ in Ptpn6 $6^{f l f l}$ mir-17-92 fl/fl Cd19Cre/ + mice (Fig. 10a,b), which could be due to the reduction of IgM antibody-producing B1 cells resulting from mir-17-92-deficiency (Fig. 2). Strikingly, antiDNA antibodies of the IgG2c isotype, which were abundantly present in Ptpn $6^{f l / f l}$ mir-17-92 $+1+C d 19^{C r e l}+$ mice, were significantly reduced in Ptpn $6^{f l / f l}$ mir-17-92 fl/fl $C d 19^{C r e /+}$ mice. Immunofluorescence staining of renal sections (Fig. 10c) revealed the presence of prominently coarse granular and segmental IgG depositions in the mesangium of the glomeruli of Ptpn $6^{f l / f l} \mathrm{mir}$ $17-92^{+1+} \mathrm{Cd} 19^{\mathrm{Cre} /+}$ mice, which was indicative of immune complex glomerulonephritis. Interestingly, the amount of IgG immune complex deposition was significantly reduced in the 
a

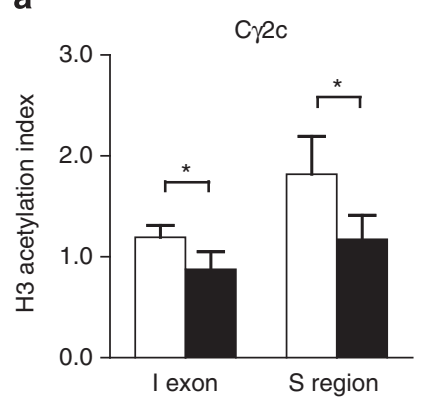

b

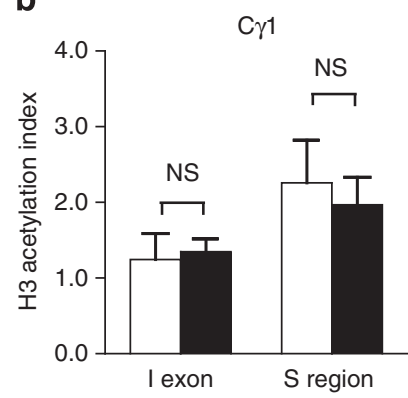

C

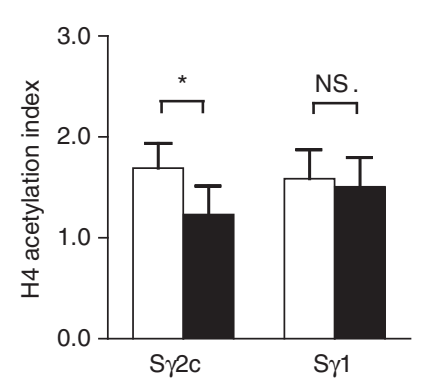

d

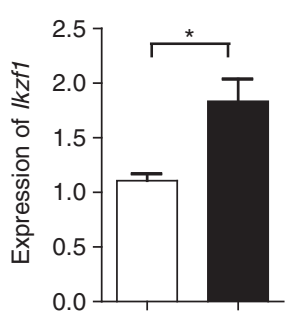

e

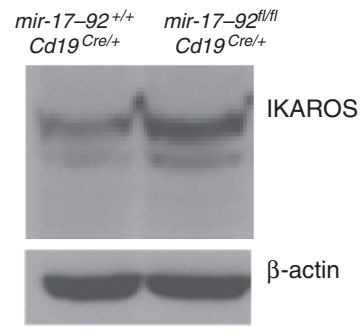

f

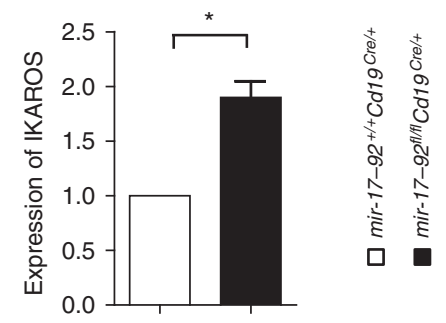

Figure 8 | Reduced histone acetylation at $\mathbf{C} \gamma \mathbf{2 c}$ locus and increased IKAROS expression in B cells lacking miR-17-92. (a) Reduced histone H3 acetylation at $C \gamma 2 c$ in the absence of mir-17-92 bKO. Mir-17-92 control and bKO B cells were stimulated with LPS + IFN $\gamma$ for 2 days and subjected to ChIP with anti-AcH3 or control antibodies. (b) Comparable histone $\mathrm{H} 3$ acetylation at $\mathrm{C} \gamma 1$ in mir-17-92 bKO control B cells. Mir-17-92 control and bKO B cells were stimulated with LPS + IL-4 for 2 days and subjected to ChIP with anti-AcH3 or control antibodies. (c) Impaired histone $\mathrm{H} 4$ acetylation at $\mathrm{S} \gamma 2 \mathrm{c}$ in the absence of mir-17-92 bKO. Mir-17-92 control and bKO B cells were stimulated with LPS + IFN $\gamma$ or LPS + IL-4 for 2 days and subjected to ChIP with anti-AcH4 or control antibodies. ChIP and input DNA was quantified by qPCR. Percentage of $\mathrm{AcH} 3$ and AcH4 enrichment was calculated as ChIP DNA relative to $10 \%$ input DNA. The $\mathrm{AcH} 3$ or $\mathrm{AcH} 4$ index was shown as the ratio of per cent histone acetylation enrichment at I exon or $\mathrm{S}$ region to that at Gapdh promoter region. (d-f) Increased IKAROS expression in mir-17-92-deficient B cells. Mir-17-92 control and bKO B cells were stimulated with LPS + IFN $\gamma$ for 2 days. The levels of Ikzf1 mRNA (d) and IKAROS protein (e) were determined by qRT-PCR and western blotting, respectively. The ratio of IKAROS protein to $\beta$-actin in mir-17-92 control and bKO B cells was calculated (f). Bar graphs represent mean $\pm s$.d. $(n=3$ for a-d; $n=5$ for $\mathbf{f})$. Data are representative of five (e) experiments. Unpaired two-tailed Student's $t$-test was used for statistical analyses. NS, not significant; ${ }^{\star} P<0.05$.

kidneys of Ptpn $6^{f l f l}$ mir-17-92 $2^{f l f l} \mathrm{Cd} 19^{\mathrm{Cre} /}+$ mice (Fig. 10c,d). These results suggest that the ablation of mir-17-92 could potentially suppress IgG2c autoantibody production and therefore alleviate symptoms of systemic autoimmunity in mice.

\section{Discussion}

We examined the role of mir-17-92 in late-stage B-cell differentiation and function by ablating it using CD19-cre. We show that mir-17-92 is not absolutely required for conventional $\mathrm{B}$-cell maturation in the periphery in contrast to its essential role in early B lymphopoiesis. However, we unveil two novel roles for mir-17-92 in peripheral B cells, namely the modulation of PC homing to the BM and selective regulation of IgG2c production. We further identify S1PR1 and IKAROS as direct physiological targets of mir-17-92 responsible for these two outcomes.

Previous studies have postulated that mir-17-92 could largely account for Dicer's function in early B-lymphoiesis by targeting $\mathrm{BIM}^{11,18}$. Here we demonstrate that mir-17-92 is largely dispensable for the maturation of conventional $\mathrm{B}$ cells in the periphery, suggesting that it plays a more important role in early $\mathrm{B}$-lymphoiesis than in peripheral B-cell maturation. However, we demonstrate that mir-17-92 is required for B1 cell development and TI immune response. We also show that BIM protein but not mRNA level is increased in mir-17-92-deficient B cells and could account for the modestly decreased total peripheral B cells in mir17-92 bKO mice. Previous studies of mir-17-92-deficient or overexpressing B cells ${ }^{11,39}$ have demonstrated either enhanced or decreased BIM protein expression in mutant B cells, but Bim mRNA level was not examined in those studies. Our results suggest that mir-17-92 regulation of BIM mainly occurs at the protein level.
In contrast to the absolute requirement for Dicer in GC response ${ }^{13}$, mir-17-92 bKO mice have normal NP-specific IgM and IgG1 antibody production and unperturbed GC during TD antibody response, suggesting that miRNAs other than mir-17-92 are important for these processes. In support of this view, a previous miRNA deep-sequencing study has shown that mir-1792 family members are not amongst the most highly expressed miRNAs in GC B cells ${ }^{40}$.

Interestingly, we show that mir-17-92 is important for modulating PC homing to BM where survival niches are provided for their long-term persistence ${ }^{41-43}$. We show that five out of six members of mir-17-92 cluster target the $3^{\prime}$ UTR of S1pr1 at four different sites and the absence of mir-17-92 leads to higher S1pr1 expression in PCs. It is possible that $S 1$ pr 1 could be synergistically and simultaneously targeted by these five miRNAs in PCs. The enhanced BM homing of PCs in mir-17-92 bKO mice can be normalized by removing one copy of $S 1$ pr 1 gene. Thus, our data suggest that mir-17-92-mediated fine-tuning of S1PR1 expression is another means to control BM homing of PCs, and this insight could be useful for the development of miRNA-based drugs to interfere with PC homing to BM survival niches in PC-related diseases such as multiple myeloma and autoimmunity.

The mir-17-92-mediated modulation of S1PR1 expression and enhanced homing of PCs in mutant mice raise the possibility that the slightly decreased peripheral $B$ cells in mutant mice might be due to increased egress of $B$ cells from secondary lymphoid organs into circulation. However, our analyses showed that the distribution of naïve $\mathrm{B}$ cells in blood and secondary lymphoid organs was comparable between mir-17-92 bKO and control mice (Supplementary Fig. 9), suggesting that the mildly increased S1PR1 level in mutant naïve B cells does not significantly shift the 


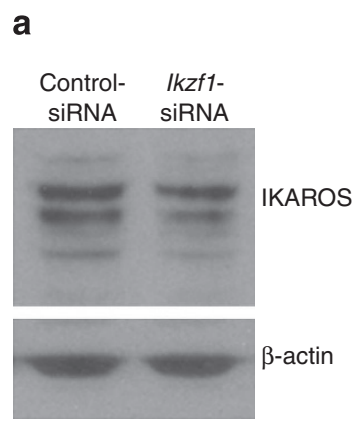

b
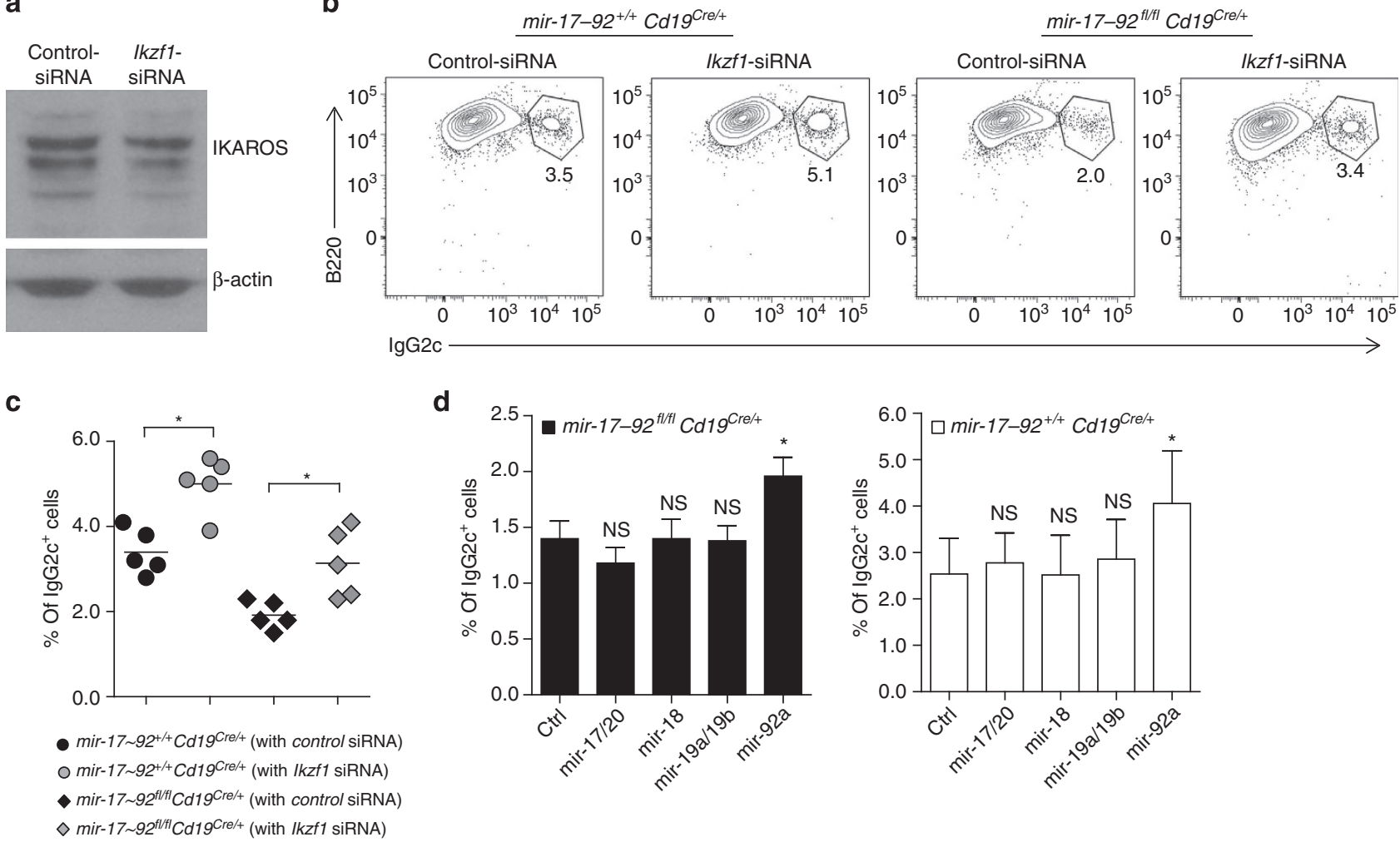

Figure 9 | MiR-92 modulates IgG2c antibody production via targeting Ikzf1. (a) Ikzf1 knockdown in mir-17-92 bKO B cells by siRNA. Mir-17-92 bKO B cells were stimulated with LPS + IFN $\gamma$ and nucleotransfected with control or Ikzf1-targeting siRNA and IKAROS protein expression was examined by western blotting. (b,c) Increased IgG2c ${ }^{+}$B cells by Ikzf1 knock-down. LPS + IFN $\gamma$-stimulated mir-17-92 control and bKO B cells were nucleotransfected with control or Ikzf1-targeting siRNAs. The presence of $\operatorname{lgG} 2 \mathrm{c}^{+} \mathrm{B}$ cells was assessed by FACS analyses (b) and their percentages were depicted in $\mathbf{c}$. (d) Increased $\operatorname{lgG} 2 \mathrm{c}^{+} \mathrm{B}$ cells resulting from mir-92 overexpression. Mir-17-92 control and bKO B cells were stimulated with LPS + IFN $\gamma$ and nucleotransfected with control or various miRNA mimics as indicated. Bar graphs represent mean \pm s.d. $(\mathbf{d}, n=5)$. Each symbol represents one mouse and horizontal lines indicate the mean (c). Data are representative of three (a) or five (b) experiments. Unpaired two-tailed Student's $t$-test was used for statistical analyses. NS, not significant; ${ }^{\star} P<0.05$.

equilibrium distribution of $\mathrm{B}$ cells in favour of blood. The noticeable homing phenotype of mutant PCs but not naïve B cells could be due to the different expression levels of mir-17-92, which is upregulated in PCs (Supplementary Fig. 10), or the differential expression of other S1PR1-targeting miRNAs in these cells.

We also show that mir-17-92 deficiency in B cells impairs IgG2c antibody production, which is accompanied by abnormal GLT and chromatin remodelling at $\mathrm{C} \gamma 2 \mathrm{a} / 2 \mathrm{c}$ locus. We further demonstrate that mir-17-92 can inhibit the expression of IKAROS, the transcription factor known to maintain repressive chromatin at $\mathrm{C} \gamma 2 \mathrm{C} / 2 \mathrm{a}$, and therefore suppress transcription, AID accessibility and CSR of IgG2a/2 $\mathrm{c}^{20}$. It was recently demonstrated that IKAROS expression in B cells could be modulated by the transcription factor IRF5 (ref. 44). Thus, our current study uncovers a post-transcriptional layer of IKAROS regulation by miRNAs. Interestingly, in IKAROS hypomorphic mice that have 10 -fold less IKAROS protein than wild-type mice ${ }^{20}$, the production of IgG2b was also enhanced. However, in our study, we did not observe a significant change in IgG2b production in mir-17-92-deficient B cells. This could be due to the mild (approximately twofold) increase in IKAROS expression in these cells. In agreement with this viewpoint, IgG2b was also not affected in IRF5-deficent B cells, which manifested modest changes in IKAROS expression ${ }^{44,45}$. It is possible that IgG2c/2a CSR is more sensitive to subtle changes in IKAROS levels than that of other Ig isotypes.
Another interesting finding from our study is that mir-17-92 deletion in $\mathrm{B}$ cells can significantly reduce the amount of pathogenic $\operatorname{IgG} 2 \mathrm{c}$ anti-DNA antibodies in autoimmune Shp1-deficient mice. In addition, IgG immune complex glomerulonephritis in Shp1-deficient mice could be attenuated by mir-17-92 deficiency. Murine IgG2c/IgG2a and its analogue isotype IgG1 in humans are the most prevalent and potent Ig isotypes found in autoimmune diseases ${ }^{46-49}$. Therefore, our results suggest that mir-17-92 could be a possible target for therapeutic intervention by providing an avenue to control pathogenic IgG2c autoantibody production in lupus.

\section{Methods}

Mice. Mir-17-92 $2^{f l f l}$, S1pr $1^{f l f l}$, Ptpn $6^{f l / f l}$ and $C d 19^{C r e /+}$ mice were obtained from The Jackson Laboratory. AicdaCre/+ mouse was generated and described previously ${ }^{13}$ and maintained in C57BL/6 background. Mir-17-92 fl/fl mice were backcrossed $>10$ generations into $\mathrm{C} 57 \mathrm{BL} / \mathrm{B}$ background before intercrossing with

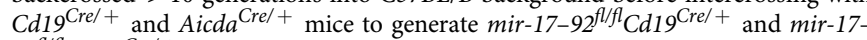
$92^{f l / f l} \mathrm{AicdaCre/+}$ mice. Genotyping of IgG2a and IgG2c alleles was performed by PCR using allele-specific primers as described ${ }^{30}$ previously. Mice of 10 to 16 weeks old of both genders were used according to the protocols approved by the Institutional Animal Care and Use Committees (IACUCs) based on guidelines from the National Advisory Committee on Laboratory Animal Research (NACLAR) of Singapore.

Immunization and FTY720 administration. Mice were immunized intraperitoneally with $100 \mu \mathrm{g}$ NP-CGG 38 (Biosearch Technologies) precipitated in Imject alum (Pierce, Biotechnology). For the in vivo inhibition of S1PR1, immunized mice were given various doses $\left(3.0,1.0\right.$ and $\left.0.3 \mathrm{mg} \mathrm{kg}^{-1}\right)$ of FTY720 (Cayman Chemical) twice by intraperitoneal injection. 


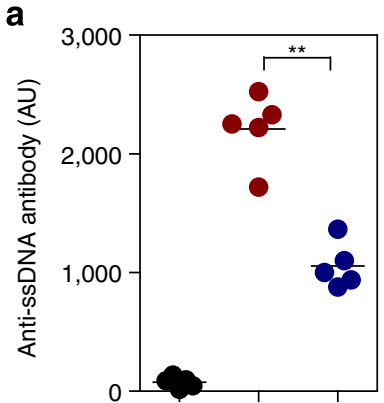

$\lg \mathrm{M}$

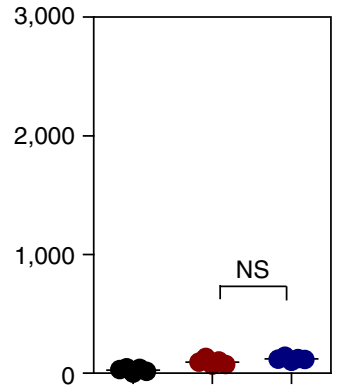

$\lg \mathrm{G} 1$

b
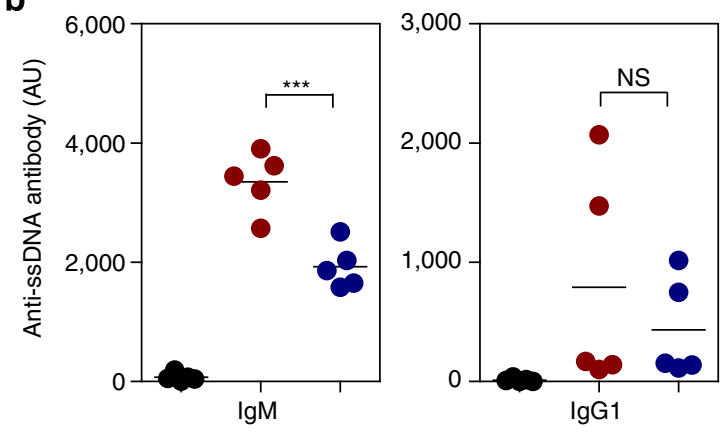

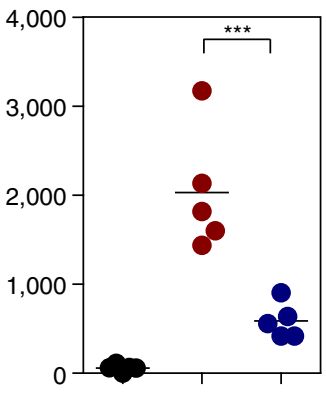

$\lg \mathrm{G} 2 \mathrm{c}$

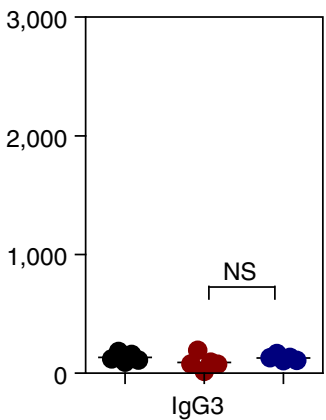

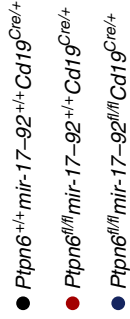
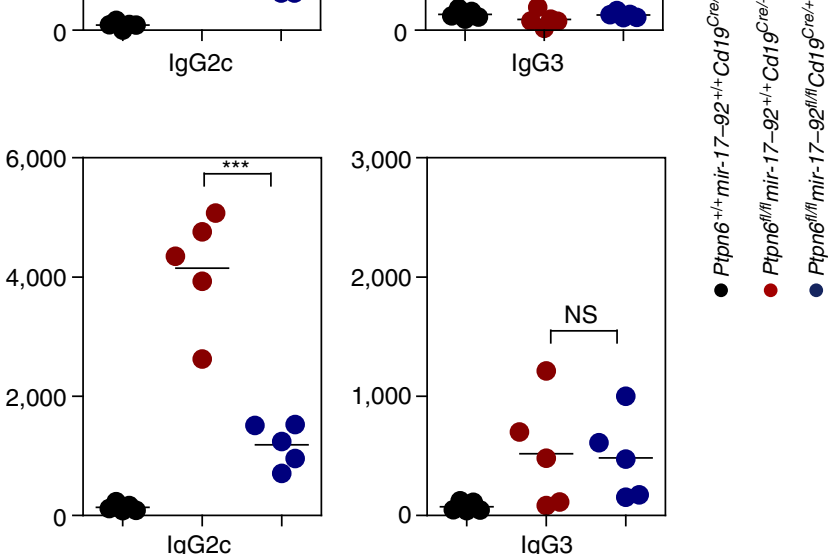

C

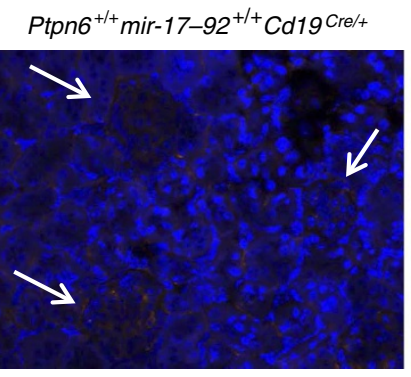

Ptpn6 ${ }^{\text {fl/fl}}$ mir-17-92 ${ }^{+/+} \mathrm{Cd} 19 \mathrm{Cre/+}$

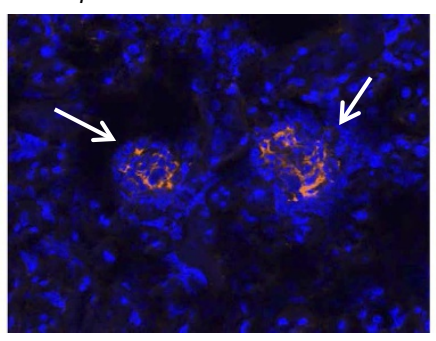

DAPI IgG

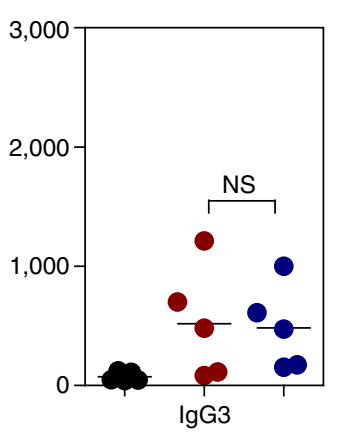

d

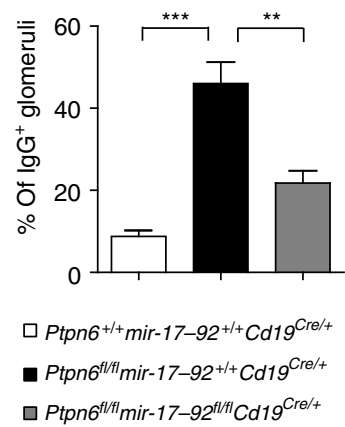

Figure 10 | Ablation of mir-17-92 in B cells attenuates autoimmunity in Shp1-deficient mice. (a,b) Sera titres of anti-ssDNA (a) and anti-dsDNA

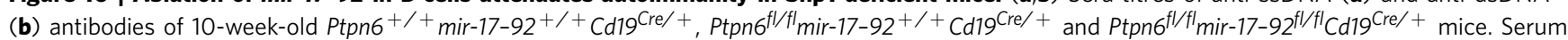
samples were assayed by ELISA for IgM, IgG1, IgG2c and IgG3 anti-DNA antibodies. (c,d) Immunofluorescence staining of renal sections of 10-week-old mice of different genotypes as indicated. Anti-mouse $\lg G$ (red) antibody was used to reveal $\lg G$ immune complexes in glomeruli (indicated by white arrows) and DAPI (blue) was used to stain DNA of renal cells (c). Scale bar, $100 \mu \mathrm{m}$. The percentages of IgG-staining positive glomeruli among $\sim 100-120$ glomeruli examined per kidney were calculated (d). Each symbol represents one mouse and small horizontal lines indicate the mean. Bar graphs represent mean \pm s.d. $(\mathbf{d}, n=5)$. Data are representative of two $(\mathbf{a}, \mathbf{b})$ and five $(\mathbf{c})$ experiments. Unpaired two-tailed Student's $t$-test was used for statistical analyses. NS, not significant; ${ }^{\star \star} P<0.01 ;{ }^{\star \star \star} P<0.001$.

Flow cytometry. Cells were harvested from BM, spleen, inguinal lymph nodes, peritoneal cavities and peripheral blood of mice and treated with Fc-block and stained with biotin- or fluorescent-labelled monoclonal antibodies (mAbs) as described ${ }^{13}$. The following mAbs were from BD Pharmingen or as specified: fluorescein isothiocyanate (FITC) anti-Fas (Cat. No: 554257), FITC anti-CD19 (Cat. No: 553785), FITC anti-IgG1 (Cat. No: 553443), FITC anti-CD21 (Cat. No: 553818), FITC-IgM (Cat. No: 553408); phycoerythrin (PE) anti-Fas (Cat. No: 554258), PE anti-CD138 (Cat. No: 553714), PE anti-B220 (Cat. No: 553090), PE anti-CD5 (Cat. No: 553023), PE anti-CD93 (ebioscience, Cat. No:12-5892); biotinylated (bio-) anti-CD38 (Cat. No: 553762), bio-anti-IgM (Cat. No: 553406), bio-anti-IgD (Cat. No: 553506), bio-anti-CD138 (Cat. No: 553717), bio-anti-CD43 (Cat. No: 553269), anti-CD62L (Cat. No: 553149), bio-anti-Thyl.2 (Cat. No: 553002), bio-anti-CD3 (Cat. No: 663060), bio-anti-Gr-1 (Cat. No: 553125), bio-anti-F4/80 (Biolegend; Cat. No:108404), bio-anti-CD5 (Cat. No:553019), bio-anti-Ter119 (Cat. No: 553612), bio-anti-DX5 (Cat. No: 553856), bio-antiNK1.1 (Cat. No: 553163), bio-anti-CD93 (Cat. No: 550434), bio-anti-IgG3 (Cat. No: 553401), bio-anti-IgG1 (Cat. No: 553441), bio-anti-IgG2c (Cat. No:553504) and bio-anti-IgG2b (Cat. No: 553393); allophycocyanin (APC) anti-CD19 (Cat. No: 550992), APC anti-CD38 (ebioscience; Cat. No:17-0381), APC-Cy7 anti-B220 (Cat. No: 552094), APC Cy7anti-CD19 (Cat. No: 557655). Biotin conjugates were visualized with Streptavidin-conjugated peridinin chlorophyll protein-cyanine 5.5
(PerCP-Cy5.5; Cat. No:551419), Streptavidin-conjugated APC (Cat. No: 554067) or APC-Cy7 (Cat. No: 554063). Fluorescein-labelled Peanut agglutinin (PNA) and 4',6-Diamidino-2-phenyindole (DAPI) were purchased from Sigma-Aldrich. PE-labelled NIP-BSA (Biosearch Technologies) were prepared using R-PE labelling kit-SH (Dojindo). All the antibodies were used in 1:100 dilution except specified elsewhere. Dead cells were excluded electronically using DAPI. Exclusion staining (Dump) for antigen-specific GC B cells or PCs included anti-IgM, anti-IgD, anti-CD138, anti-CD3, anti-Th1.2, anti-Gr-1, anti-F4/80, anti-Ter119, anti-Dx5, anti-NK1.1, anti-AA4.1 and anti-CD5 mAbs; or anti-IgD, anti-IgM, anri-CD4, anti-CD8, anti-F4/80 and anti-Gr-1 mAbs. Cell surface staining of S1PR1 was performed using rat anti-S1PR1 antibody (R\&D System) $)^{50}$. In brief, cells were stained with rat anti-S1PR1 $(5 \mu \mathrm{g} / \mathrm{ml})$ followed by donkey anti-rat IgG biotin and streptavidin-APC-Cy7. Data were collected on LSR II flow cytometer (BD Pharmingen) and analysed with Flowjo (Treestar).

Cell sorting and qRT-PCR. For cell isolation, pro-B/pre-B $\left(\mathrm{IgM}^{-} \mathrm{B} 220^{+}\right)$, immature $\mathrm{B}\left(\mathrm{IgM}^{+} \mathrm{B} 220^{+}\right)$and mature $\mathrm{B}\left(\mathrm{IgM}^{+} \mathrm{B} 220^{h i}\right)$ cells from $\mathrm{BM}$ and transitional B $\left(\mathrm{B} 220^{+} \mathrm{AA} 4.1^{+}\right)$, follicular B $\left(\mathrm{B} 220^{+} \mathrm{AA} 4.1^{-} \mathrm{CD} 23^{+} \mathrm{CD} 21^{-}\right)$, marginal zone B $\left(\mathrm{B} 220^{+} \mathrm{AA} 4.1^{-} \mathrm{CD} 23^{-} \mathrm{CD} 21^{+}\right)$and total B $\left(\mathrm{IgM}^{+} \mathrm{B} 220^{+}\right)$cells from spleens of unchallenged mice and GC B $\left(\mathrm{CD} 19^{+} \mathrm{CD} 38^{-} \mathrm{Fas}^{+}\right)$, non-GC B 
$\left(\mathrm{CD} 19^{+} \mathrm{CD}_{3}{ }^{+} \mathrm{Fas}^{-}\right)$cells and PCs $\left(\right.$Dump $\left.{ }^{-} \mathrm{B} 220^{\mathrm{lo} /}-\mathrm{CD} 138^{+} \mathrm{NIP}^{+}\right)$from spleens of immunized mice were sorted directly into Trizol LS with FACSAria (BD Pharmingen). Total RNA was prepared from sorted cells and subjected to cDNA synthesis with reverse transcriptase using random hexamers as primers (Research Instruments). Platinum SYBR Green Supermix (Life Technologies) was used in quantitative real-time PCR (qRT-PCR) for Bim, S1prl and Ikzfl using primers as follow: Bim (forward: $5^{\prime}$-CGACAGTCTCAGGAGGAACC- ${ }^{\prime}$, reverse: $5^{\prime}$-CAATG CCTTCTCCATACCAGA-3'), S1prl (forward: 5'-GAGTCCTGCGGACGCAG CTT- $3^{\prime}$, reverse: $5^{\prime}$-AGCCAGGTCAGCGAGCAATCC- $3^{\prime}$ ) and $I k z f 1$ ( forward: $5^{\prime}$-GGATCATTCTTGGCCCCCAAA- $3^{\prime}$, reverse: $5^{\prime}$-ACCCTCATCGACATCCAT TGT-3'). TaqMan MicroRNA system (Life Technologies) was used for miRNA quantification. GLT levels for $\gamma 3, \gamma 1, \gamma 2 c$ and $\gamma 2 b$ were determined as described ${ }^{20}$ previously.

Immunofluorescence histology. Splenic and renal sections of $8 \mu \mathrm{m}$ thickness were prepared from tissues frozen in Tissue-Tek (Sakura) as described previously ${ }^{13}$. After blocking, sections were stained at room temperature for $30 \mathrm{~min}$ with a cocktail of antibodies and mounted with CYTOSEAL 60 (Electron Microscopy Sciences) and analysed on a FV1000 confocal microscope (Olympus).

ELISA and ELISPOT assay. Sera were collected from unchallenged or immunized mice on various days. Total or NP-specific antibodies of various isotypes in sera were measured via enzyme-linked immunosorbent assay (ELISA). The relative titres of NP-specific serum antibodies are shown as the relative of dilutions that give absorbance reading within the linear range of the assay ${ }^{51}$. The frequency of NP-specific IgG1 antibody-secreting cells was determined by ELISPOT assay ${ }^{13}$.

Plasmid construction and luciferase reporter assay. Mir-17, mir-18a, mir-19a, mir-19b, mir-20a, mir-92a and mir-17-92 cluster were cloned into MSCV retroviral vector. The $3^{\prime}$ UTR fragments of mouse SIprl and $I k z f 1$ with predicted binding sites for individual miRNAs and their mutants were amplified from genomic DNA and inserted into pmirGLO plasmid (Promega) and confirmed by sequencing. For luciferase reporter assay, HEK 293 cells were plated into 48 -well plates at $1 \times 10^{5}$ cells per well $16 \mathrm{~h}$ before transfection. pmirGLO $(0.2 \mu \mathrm{g})$ reporter plasmid containing the S1prl and Ikzf1 $3^{\prime}$ UTR fragments or their mutants and $0.2 \mu \mathrm{g}$ different miRNA-expressing plasmids were transfected into each well using Lipofectamine 2000 transfection reagent (Invitrogen) in triplicate. Luciferase assays were performed $48 \mathrm{~h}$ after transfection using the Dual-Luciferase Reporter Assay System (Promega) on a SpectraMax Luminescence Microplate Reader (Molecular Devices). Luciferase activity was expressed as ratio between firefly and renilla luciferase activities.

Cell culture and nucleofection of B cells. Splenic B cells were purified with anti$\mathrm{CD} 43 \mathrm{mAb}$-coupled magnetic beads using magnetic-activated cell sorting negative selection protocol (Miltenyi Biotech, Bergisch Gladbach, Germany). Cells were stimulated for 3 days with $10 \mu \mathrm{g} \mathrm{ml}^{-1}$ LPS (Sigma) in DMEM supplemented with the necessary additives. IL-4 $\left(10 \mathrm{ng} \mathrm{ml}^{-1}\right)$, IL-5 $\left(5 \mathrm{ng} \mathrm{ml}^{-1}\right)$, IFN $\gamma\left(10 \mathrm{ng} \mathrm{ml}^{-1}\right)$ and $\operatorname{TGF} \beta\left(2 \mathrm{ng} \mathrm{ml}^{-1}\right)$ from R\&D Systems were used to induce preferential Ig class switching to various isotypes. For nucleofection, purified B cells were stimulated with $10 \mu \mathrm{g} \mathrm{ml}^{-1}$ LPS and $10 \mathrm{ng} \mathrm{ml}^{-1}$ IFN $\gamma$ overnight and transfected with siRNA or miRNA mimics from Life Technologies using P4 Primary Cell 4D-Nucleofector $\mathrm{X}$ Kit on an Amaxa 4D-nucleofector (Lonza). Transfected cells were recultured and analysed $48 \mathrm{~h}$ later.

Western blotting. Cells were lysed and protein concentrations were determined as described previously ${ }^{52}$. Equal amount of proteins was loaded and subjected to SDS-polyacrylamide gel electrophoresis, transferred and blotted with specific antibodies. BIM-specific antibody (C34C5) was from Cell Signalling. The IKAROSspecific antibody (M20) was from Santa Cruz. Western blot images shown in Figs 1, 8 and 9 have been cropped for presentation. Full-sized images are presented in Supplementary Fig. 11.

ChIP-qPCR. ChIP was performed using EZ-ChIP kit (Millipore) according to the manufacturer's protocol. In brief, $1 \times 10^{7}$ stimulated B cells were crosslinked in $1 \%$ formaldehyde. Chromatin was sonicated to $500-1,000 \mathrm{bp}$ using Misonix Sonicator 3000 Ultrasonic Cell Disruptor (QSonica). Sonicated chromatin was precleared with Protein G Agarose and subsequently subjected to immunoprecipitation with anti-H3Ac, anti-H4Ac or control antibodies (Millipore). One per cent of the precleared chromatin was saved as input. Protein/DNA complexes were eluted and reverse crosslinked to free DNA, which was further purified using spin columns (Millipore). The input DNA was diluted 10 times. Two $\mu \mathrm{l}$ of DNA samples was analysed by qPCR using $I \gamma 1, S \gamma 1, I \gamma 2 c, S \gamma 2 c$ and Gapdh primers ${ }^{20,53}$. The acetylation index was calculated described previously ${ }^{20}$.

Statistics. Unpaired two-tailed Student's $t$-test was used for statistical analyses.

\section{References}

1. Rajewsky, K. Clonal selection and learning in the antibody system. Nature 381, 751-758 (1996).

2. Goodnow, C. C., Vinuesa, C. G., Randall, K. L., Mackay, F. \& Brink, R. Control systems and decision making for antibody production. Nat. Immunol. 11, 681-688 (2010).

3. Nutt, S. L. \& Kee, B. L. The transcriptional regulation of B cell lineage commitment. Immunity 26, 715-725 (2007).

4. Mandel, E. M. \& Grosschedl, R. Transcription control of early B cell differentiation. Curr. Opin. Immunol. 22, 161-167 (2010).

5. Xiao, C. \& Rajewsky, K. MicroRNA control in the immune system: basic principles. Cell 136, 26-36 (2009).

6. de Yebenes, V. G., Bartolome-Izquierdo, N. \& Ramiro, A. R. Regulation of B-cell development and function by microRNAs. Immunol. Rev. 253, 25-39 (2013).

7. Lee, Y. et al. MicroRNA genes are transcribed by RNA polymerase II. EMBO J. 23, 4051-4060 (2004).

8. Lee, Y. et al. The nuclear RNase III Drosha initiates microRNA processing. Nature 425, 415-419 (2003).

9. Hutvagner, G. et al. A cellular function for the RNA-interference enzyme Dicer in the maturation of the let-7 small temporal RNA. Science 293, 834-838 (2001).

10. Lewis, B. P., Burge, C. B. \& Bartel, D. P. Conserved seed pairing, often flanked by adenosines, indicates that thousands of human genes are microRNA targets. Cell 120, 15-20 (2005).

11. Koralov, S. B. et al. Dicer ablation affects antibody diversity and cell survival in the B lymphocyte lineage. Cell 132, 860-874 (2008).

12. Belver, L., de Yebenes, V. G. \& Ramiro, A. R. MicroRNAs prevent the generation of autoreactive antibodies. Immunity 33, 713-722 (2010).

13. Xu, S., Guo, K., Zeng, Q., Huo, J. \& Lam, K. P. The RNase III enzyme Dicer is essential for germinal center B-cell formation. Blood 119, 767-776 (2012).

14. Ota, A. et al. Identification and characterization of a novel gene, C13orf25, as a target for 13q31-q32 amplification in malignant lymphoma. Cancer Res. 64, 3087-3095 (2004).

15. Tagawa, H. \& Seto, M. A microRNA cluster as a target of genomic amplification in malignant lymphoma. Leukemia 19, 2013-2016 (2005).

16. He, L. et al. A microRNA polycistron as a potential human oncogene. Nature 435, 828-833 (2005).

17. Jin, H. Y. et al. MicroRNA-17 92 plays a causative role in lymphomagenesis by coordinating multiple oncogenic pathways. EMBO J. 32, 2377-2391 (2013)

18. Ventura, A. et al. Targeted deletion reveals essential and overlapping functions of the miR-17 through 92 family of miRNA clusters. Cell 132, 875-886 (2008).

19. Kabashima, K. et al. Plasma cell S1P1 expression determines secondary lymphoid organ retention versus bone marrow tropism. J. Exp. Med. 203, 2683-2690 (2006).

20. Sellars, M., Reina-San-Martin, B., Kastner, P. \& Chan, S. Ikaros controls isotype selection during immunoglobulin class switch recombination. J. Exp. Med. 206, 1073-1087 (2009).

21. Mavrakis, K. J. et al. A cooperative microRNA-tumor suppressor gene network in acute T-cell lymphoblastic leukemia (T-ALL). Nat. Genet. 43, 673-678 (2011).

22. Schmidt-Supprian, M. \& Rajewsky, K. Vagaries of conditional gene targeting. Nat. Immunol. 8, 665-668 (2007).

23. Baumgarth, $\mathrm{N}$. The double life of a B-1 cell: self-reactivity selects for protective effector functions. Nat. Rev. Immunol. 11, 34-46 (2011).

24. McHeyzer-Williams, L. J. \& McHeyzer-Williams, M. G. Antigen-specific memory B cell development. Annu. Rev. Immunol. 23, 487-513 (2005).

25. Tarlinton, D., Radbruch, A., Hiepe, F. \& Dorner, T. Plasma cell differentiation and survival. Curr. Opin. Immunol. 20, 162-169 (2008).

26. Nie, Y. et al. The role of CXCR4 in maintaining peripheral B cell compartments and humoral immunity. J. Exp. Med. 200, 1145-1156 (2004).

27. Cyster, J. G. \& Schwab, S. R. Sphingosine-1-phosphate and lymphocyte egress from lymphoid organs. Annu. Rev. Immunol. 30, 69-94 (2012).

28. Chiba, K. et al. FTY720, a novel immunosuppressant, induces sequestration of circulating mature lymphocytes by acceleration of lymphocyte homing in rats. I. FTY720 selectively decreases the number of circulating mature lymphocytes by acceleration of lymphocyte homing. J. Immunol. 160, 5037-5044 (1998).

29. Brinkmann, V. et al. The immune modulator FTY720 targets sphingosine 1-phosphate receptors. J. Biol. Chem. 277, 21453-21457 (2002).

30. Zhang, Z., Goldschmidt, T. \& Salter, H. Possible allelic structure of IgG2a and IgG2c in mice. Mol. Immunol. 50, 169-171 (2012).

31. Stavnezer, J., Guikema, J. E. \& Schrader, C. E. Mechanism and regulation of class switch recombination. Annu. Rev. Immunol. 26, 261-292 (2008).

32. Nambu, Y. et al. Transcription-coupled events associating with immunoglobulin switch region chromatin. Science 302, 2137-2140 (2003).

33. Wang, L., Whang, N., Wuerffel, R. \& Kenter, A. L. AID-dependent histone acetylation is detected in immunoglobulin S regions. J. Exp. Med. 203, 215-226 (2006). 
34. Blanco, F. et al. IgG subclasses in systemic lupus erythematosus and other autoimmune rheumatic diseases. Lupus 1, 391-399 (1992).

35. Tiikkainen, U., Wangel, A., Appleton, S. L. \& Arthur, D. Subclasses of IgG anticardiolipin antibodies in patients with systemic lupus erythematosus. Scand. J. Immunol. 34, 265-271 (1991).

36. Martin, F. \& Chan, A. C. Pathogenic roles of B cells in human autoimmunity; insights from the clinic. Immunity 20, 517-527 (2004).

37. Yung, S. \& Chan, T. M. Anti-DNA antibodies in the pathogenesis of lupus nephritis--the emerging mechanisms. Autoimmun. Rev. 7, 317-321 (2008).

38. Pao, L. I. et al. B cell-specific deletion of protein-tyrosine phosphatase Shp1 promotes B-1a cell development and causes systemic autoimmunity. Immunity 27, 35-48 (2007).

39. Xiao, C. et al. Lymphoproliferative disease and autoimmunity in mice with increased miR-17-92 expression in lymphocytes. Nat. Immunol. 9, 405-414 (2008).

40. Kuchen, S. et al. Regulation of microRNA expression and abundance during lymphopoiesis. Immunity 32, 828-839 (2010).

41. Sze, D. M., Toellner, K. M., Garcia,d, V., Taylor, D. R. \& MacLennan, I. C. Intrinsic constraint on plasmablast growth and extrinsic limits of plasma cell survival. J. Exp. Med. 192, 813-821 (2000).

42. Cassese, G. et al. Plasma cell survival is mediated by synergistic effects of cytokines and adhesion-dependent signals. J. Immunol. 171, 1684-1690 (2003).

43. Minges Wols, H. A., Underhill, G. H., Kansas, G. S. \& Witte, P. L. The role of bone marrow-derived stromal cells in the maintenance of plasma cell longevity. J. Immunol. 169, 4213-4221 (2002).

44. Fang, C. M. et al. Unique contribution of IRF-5-Ikaros axis to the B-cell IgG2a response. Genes Immun. 13, 421-430 (2012).

45. Savitsky, D. A., Yanai, H., Tamura, T., Taniguchi, T. \& Honda, K. Contribution of IRF5 in B cells to the development of murine SLE-like disease through its transcriptional control of the IgG2a locus. Proc. Natl Acad. Sci. USA 107, 10154-10159 (2010)

46. Coutelier, J. P., van der Logt, J. T., Heessen, F. W., Warnier, G. \& Van Snick, J. IgG2a restriction of murine antibodies elicited by viral infections. J. Exp. Med. 165, 64-69 (1987).

47. Kipps, T. J., Parham, P., Punt, J. \& Herzenberg, L. A. Importance of immunoglobulin isotype in human antibody-dependent, cell-mediated cytotoxicity directed by murine monoclonal antibodies. J. Exp. Med. 161, 1-17 (1985).

48. Nimmerjahn, F. \& Ravetch, J. V. Divergent immunoglobulin g subclass activity through selective Fc receptor binding. Science 310, 1510-1512 (2005).
49. Fossati-Jimack, L. et al. Markedly different pathogenicity of four immunoglobulin $\mathrm{G}$ isotype-switch variants of an antierythrocyte autoantibody is based on their capacity to interact in vivo with the low-affinity Fcgamma receptor III. J. Exp. Med. 191, 1293-1302 (2000).

50. Arnon, T. I. et al. GRK2-dependent S1PR1 desensitization is required for lymphocytes to overcome their attraction to blood. Science 333, 1898-1903 (2011).

51. Ye, B. H. et al. The BCL-6 proto-oncogene controls germinal-centre formation and Th2-type inflammation. Nat. Genet. 16, 161-170 (1997).

52. Xu, S., Huo, J., Lee, K. G., Kurosaki, T. \& Lam, K. P. Phospholipase Cgamma2 is critical for Dectin-1-mediated Ca2 + flux and cytokine production in dendritic cells. J. Biol. Chem. 284, 7038-7046 (2009).

53. Matsui, T. et al. Proviral silencing in embryonic stem cells requires the histone methyltransferase ESET. Nature 464, 927-931 (2010).

\section{Acknowledgements}

We thank Kenny Boey for the maintenance of mice and Ke Guo for helpful suggestions with histology. This work was funded by the Biomedical Research Council of the Singapore Agency for Science Technology and Research.

\section{Author contributions}

S.X. and K.-P. L conceptualized the research and wrote the manuscript; S.X., X.O. and K.-P. L. designed the experiments and analysed the data. S.X., X.O., J.H., K.L., Y.H. and S.C. performed the experiments. All authors discussed the results and commented on the manuscript.

\section{Additional information}

Supplementary Information accompanies this paper at http://www.nature.com/ naturecommunications

Competing financial interests: The authors declare no competing financial interests.

Reprints and permission information is available at http://npg.nature.com/ reprintsandpermissions/

How to cite this article: $\mathrm{Xu}, \mathrm{S}$. et al. Mir-17-92 regulates bone marrow homing of plasma cells and production of immunoglobulin G2c. Nat. Commun. 6:6764 doi: 10.1038/ncomms7764 (2015). 